\title{
ОПРЕДЕЛЕНИЕ ОПТИМАЛЬНОЙ ПРЕМИИ В СДЕЛКАХ СЛИЯНИЯ И ПОГЛОЩЕНИЯ В НЕФТЕГАЗОВОМ СЕКТОРЕ
}

\author{
В.В. Назарова ${ }^{1}$, О.Р. Шевякина ${ }^{2}$
}

ᄃㄷㄹки слияний и поглощений являются важной частью экономики любой страны. По цивилизоح ванности проведения сделок и по состоянию рынка слияний и поглощений можно судить о раз-витии страны в целом. В сложных условиях компаниям все труднее проводить успешные сделки M\&A. Необходимо учитывать большое количество нюансов на стадии подготовки к сделке, которые оказывают непосредственное влияние на качество ее проведения. Одним из самых важных  шсодержит очень серьезную основу для понимания верного направления заключения сделки M\&A, Iтак как от этого зависит, выгодно ли будет исполнена сделка, будет ли получен положительный синергетический эффект и многие другие важные аспекты. Говоря в общем, если компания-поглотитель вовремя не рассчитает фактически верную величину премии, то может оказаться в убытке, отдав за сделку большую стоимость, чем получит от нее прибыли.

Актуальность данного исследования заключается в том, что на стадии подготовки сделки М\&А ( ) необходимо определить именно оптимальную и верную величину премии, как для продавца, так и 乞для покупателя. Для продавца важным становится получить за сделку сумму, не меньшую стоимости той части активов, которую он продает, или всей стоимости компании. Для покупателя важно -не переплатить за сделку, поскольку слишком высокий показатель премии гарантирует отсутствие ๓в будущем синергетических эффектов, а возможно, отрицательный исход для компании вплоть до ғее банкротства.

ol Цель данной статьи заключается в определении формирования оптимальной величины премии в сделках М\&A через идентификацию факторов, оказывающих непосредственное влияние на показатель премии.

Объектом настоящего исследования являются сделки M\&A в нефтегазовой отрасли. Предмет - премии, уплачиваемые в ходе сделки M\&A в нефтегазовом секторе покупателем продавцу.

В результате проведения исследования была создана регрессионная модель, объясняющая зависимость величины премии от некоторых финансовых и нефинансовых факторов: рыночная капитализация положительно влияет на образование величины премии; операционная прибыль компаниимишени и количество размещенных акций находятся в отрицательной зависимости от величины премии; при конгломератных и вертикальных (вертикальных - немного ниже) слияниях величина премии значительно выше, чем при горизонтальном слиянии; информационная асимметрия положительно влияет на премию; трансграничный тип сделки также оказывает положительное влияние на величину премии. Итоговая модель имеет хорошую объясняющую способность

Необходимо также отметить, что не только данные финансовые показатели компании-цели оказывают влияние на величину премии, но и связанные с ними показатели, которые могут быть использованы при расчетах.

Ключевые слова: слияние и поглощение, стоимость компании, премия при сделках слияния и поглощения, нефтегазовый сектор, эконометрическая регрессионная многофакторная модель

JEL: L1

На сегодняшний день упоминания о сделках слияний и поглощений встречаются регулярно. В современном экономическом мире сложилась ситуация, при которой фирмам и компаниям необходимо бороться за место на рынках путем реформирования политики собственной деятельности или реорганизации структуры компании. Эти обстоятельства чаще всего проявляются в неспособности к конкуренции на рынке, падении ликвидности активов, уменьшении прибыли вследствие какихлибо макроэкономических событий или ситуаций внутри компании. Именно стратегия слияния и поглощения является одним из надежных путей решения таких проблем.

1. В.В. Назарова, канд. эконом. наук, доцент департамента финансов СПбШЭиМ.

2. О.Р. Шевякина, стажер-исследователь выпускница Бакалаврской программы «Экономика» СПбШЭиМ 
Сделки слияний и поглощений (M\&A, или Mergers and Acquisitions) являются важной частью экономики страны. По ситуации на рынке M\&A и по уровню цивилизованности проведения сделок можно судить об экономическом развитии государства. Российский рынок M\&A успешно прошел стадию формирования за последние 10 лет, несмотря на то что в последние два года и на данный

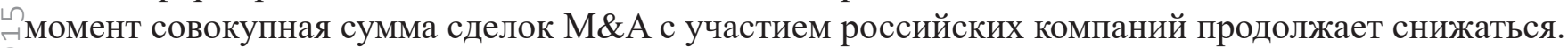

Мировой рынок слияний и поглощений за последние несколько лет имеет довольно высокие по矛 казатели. Несмотря на это, ситуация на российском рынке M\&A идет вразрез с мировым тенденциями. Из-за политического и экономического кризиса 2014 год для России явился годом сложных испытаний. Уменьшение объемов рынка - следствие введения санкций из-за сложной обстановки на Украине, обесценения рубля, падения цен на нефть.

По оценкам экспертов КПМГ, 2015 год станет для России годом сокращения объемов рынка M\&A, ऽуменьшения крупных сделок. Несмотря на эти факторы, отрицательно сказывающиеся на рынке слияний и поглощений, российская экономика нацелена на долгосрочное развитие экономического потенциала сделок М\&A.

В связи со сложной экономической и политической обстановкой компаниям все труднее проводить успешные сделки M\&A. Необходимо на стадии подготовки к сделке учитывать большое количе乞ство нюансов, которые оказывают непосредственное влияние на качество ее проведения: опредеоление достоверных мотивов покупателя и продавца; тщательное изучение финансовой истории и -настоящего положения компании-мишени во избежание переплаты и получения отрицательного ๓синергетического эффекта; составление четкого плана. Внимательно надо проводить саму сделку $\checkmark$ и развивать компанию по итогам завершения сделки, то есть после нее создавать стоимость компа文нии; верно взаимодействовать с объектом приобретения. Такие же выверенные действия необхоч

ППроблема, рассматриваемая в данной статье, заключается в том, что одним из самых важных ню๑ансов сделки M\&A является определение оптимальной величины премии. Данный вопрос - основа для понимания верного направления заключения сделки M\&A, так как от этого зависит, выгодно ли будет исполнена сделка, будет ли получен положительный синергетический эффект, и многие другие важные аспекты. Говоря в общем, если компания-поглотитель вовремя не рассчитает фактически верную величину премии, то может оказаться в убытке, отдав за сделку большую премию, чем получит прибыли.

Актуальность данной проблемы заключается в том, что на стадии подготовки сделки М\&А необходимо определить именно оптимальную и верную величину премии как для продавца, так и для покупателя. Для продавца важным становится получить за сделку сумму, не меньшую стоимости той части активов, которую он продает, или всей стоимости компании. Для покупателя важно не переплатить за сделку, поскольку слишком высокий показатель премии гарантирует отсутствие в будущем синергетических эффектов, а возможно, отрицательный исход для компании, вплоть до ее банкротства.

Для исследования данного вопроса была выбрана нефтегазовая отрасль, поскольку она характеризуется высоким уровнем развития и составляет важнейшую часть экономики. Также данная отрасль ввиду своих особенностей интересна для рассмотрения.

Объектом настоящего исследования являются сделки M\&A в нефтегазовой отрасли. Предмет исследования - премии, уплаченные в ходе сделки M\&A в нефтегазовом секторе покупателем продавцу.

В процессе подготовки и планирования сделки компания-покупатель рассчитывает получить ожидаемые синергетические выгоды, а также выплачивает премию за акции компании-мишени. Чистая стоимость поглощения, или $N A V$, рассчитывается по следующей формуле:

$N A V=V A B-[V A+V B]-A P R-E$

где:

$V A B \equiv$ стоимость компании после объединения; 
$V B \equiv$ рыночная стоимость акций компании $B$;

$A P R \equiv$ премия, выплаченная компанией А за компанию $B$;

$E \equiv$ издержки процесса слияния и поглощения;

ๆ $V A \equiv$ оценка компанией А своей собственной стоимости.

Преобразовав формулу (1), получаем:

I $N A V=[V A B-(V A+V B)]-(A P R+E)$.

При расчете положительной чистой стоимости поглощения необходимо учесть, что часть формулы,

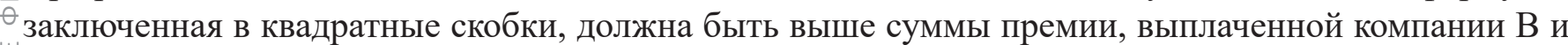
乞издержек сделки. Если получается, что эта часть имеет более низкое значение, то можно сделать вывод о том, что компания-покупатель переплатила за сделку.

Непосредственно переходя к анализу нефтегазовой отрасли, необходимо отметить тенденции рынка слияний и поглощений.

2014 год показал спад активности на рынке слияний и поглощений в нефтегазовом секторе из-за () экономических санкций и падения цен на нефть. Так, объем сделок в 2014 году составил 20,6 млрд пдолларов США против 26,9 млрд долларов США в 2013 году [KPMG]. Рынок сократился практически на 24\%. Но все же количество сделок увеличилось: в 2013 году - 38 транзакции, а в 2014 году -52 (см. диаграмму 1).

\section{Общая стоимость и количество сделок М\&A в нефтегазовом секторе}

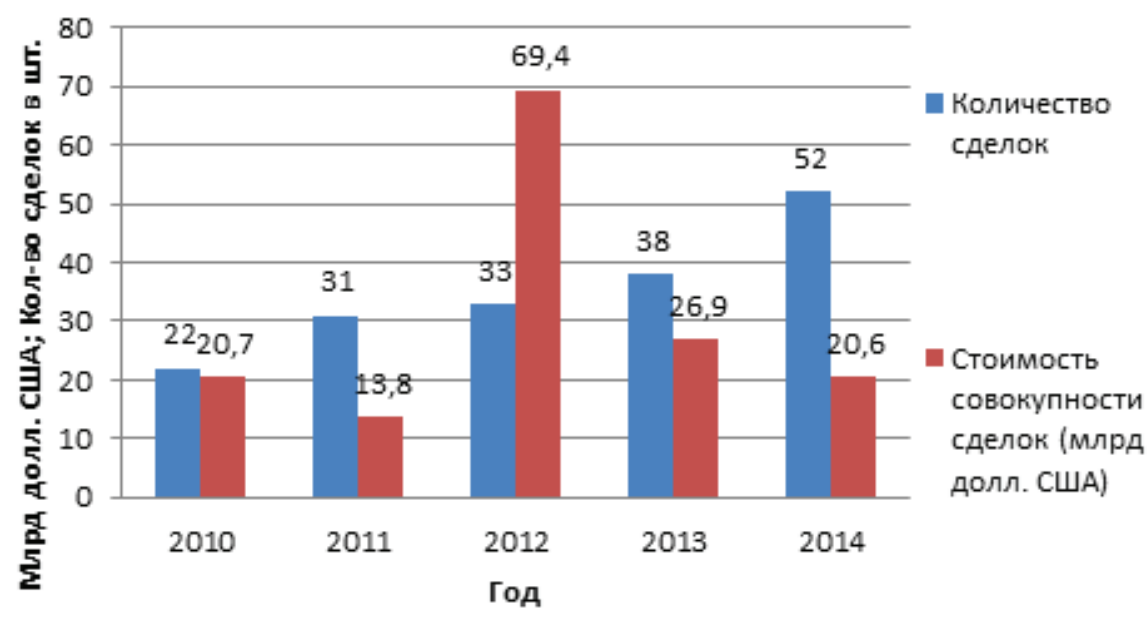

Диаграмма 1. Динамика стоимости и количества сделок М\&A в нефтегазовом секторе в России Источник: [KPMG].

Что касается ценового аспекта сделок, то средняя цена за сделку в данной отрасли, даже исключая из статистики мегасделки, равна 213,7 млн долл. США против 117,2 млрд долл. США в прошлом, 2013 году. Соответственно, нефтегазовая отрасль остается самой дорогой на российском рынке M\&A [KPMG; Bloomberg].

Несмотря на все трудности, обрушившиеся на нефтегазовый сектор, именно в нем было проведено пять из 12 мегасделок (сделки, стоимость которых превышает 1 млрд долл. США) на всем рынке M\&A.

Доступ к зарубежному финансированию для российских компаний в настоящее время ограничен. Согласно общей тенденции рынка M\&A, преобладающий вид сделок в нефтегазовой отрасли внутренние сделки. Всего лишь $9 \%$ от совокупной стоимости сделок включают покупку россий- 
ских активов иностранными инвесторами (см. диаграмму 2), то есть на данный момент российские компании инвестиционно непривлекательны для зарубежных. Несмотря на это, количество сделок такого вида составляет практически $20 \%$ от общего количества сделок на российском рынке (см. диаграмму 3).

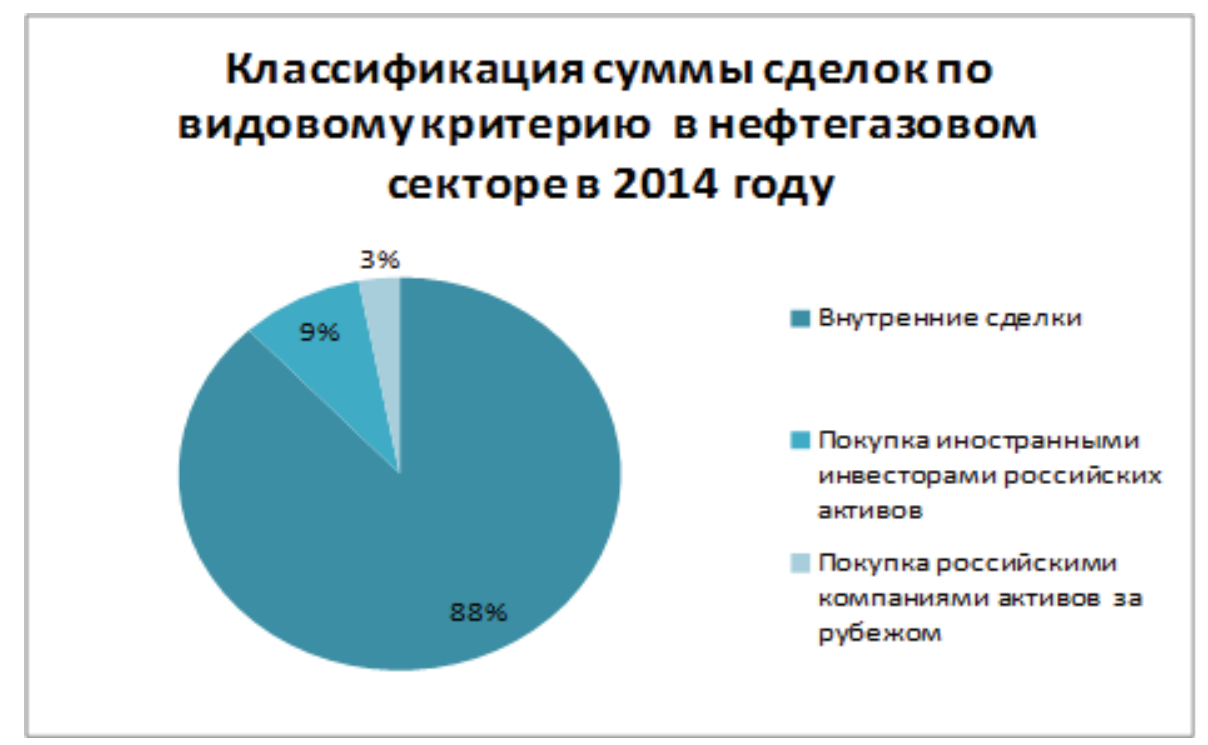

Диаграмма 2. Сумма сделок по видам в нефтегазовом секторе

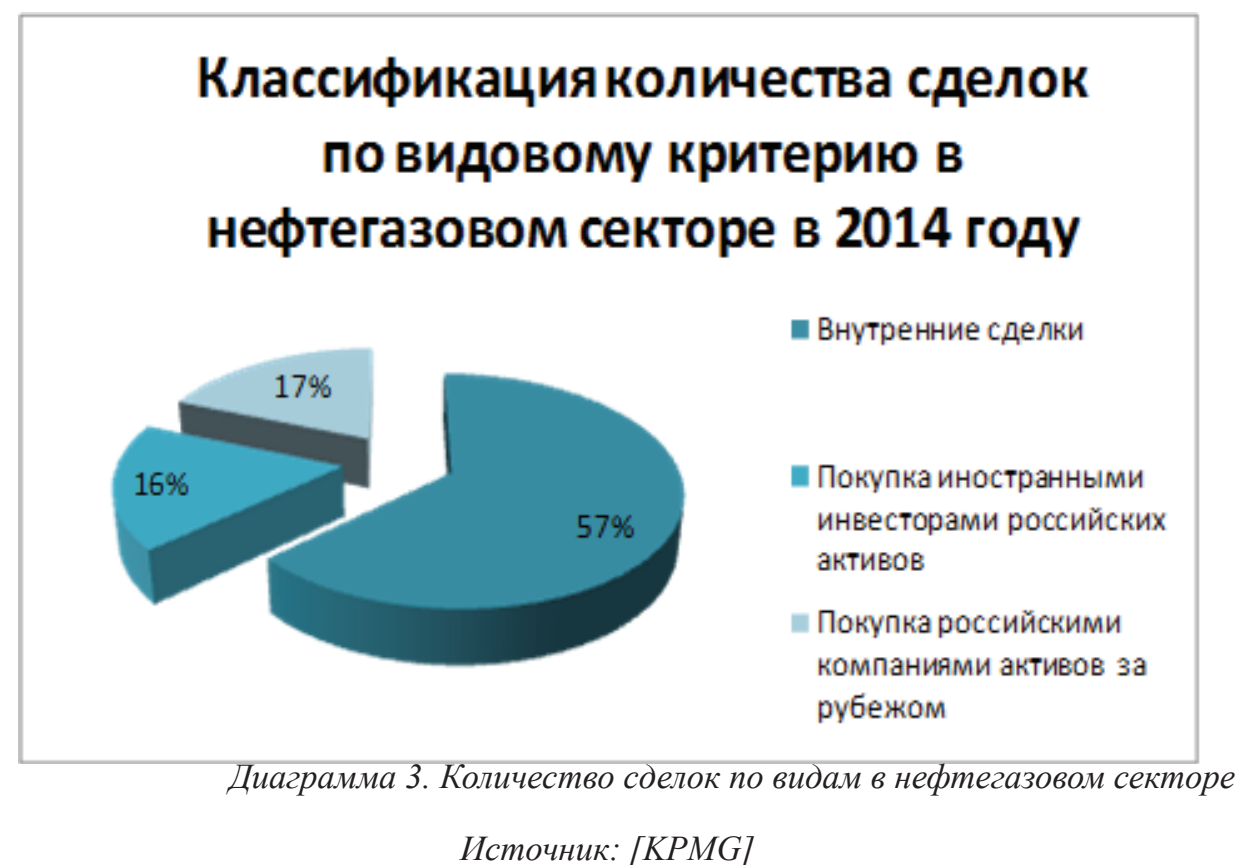

Что касается мирового рынка M\&А в нефтегазовом секторе, по сравнению с 2013 годом в 2014 году совокупная стоимость сделок M\&А выросла на 69\% и составила 443 млрд долларов США в денежном эквиваленте, но количество сделок сократилось практически на $20 \%$.

На сегодняшний момент очевиден тот факт, что дальнейшая активность на рынке М\&А в нефтегазовом секторе будет определяться уровнем цен на нефть. Также имеет место предположение о том, что большой процент нефтегазовых компаний с низким уровнем хеджирования рисков, а также с высокой долговой нагрузкой рискует стать мишенью для компаний с более устойчивым финансовым положением. Остается только надеяться, что рынок M\&A в нефтегазовом секторе стабилизируется во второй половине 2015 года вследствие адаптации компаний к низким ценам на нефть.

Далее прежде чем перейти непосредственно к понятию премии при сделках слияния и поглощения, необходимо проанализировать различные виды стоимости компании, а также подходы к оценке. Данные понятия непосредственно связаны с вопросом формирования премии.

Для начала необходимо остановиться на справедливой рыночной стоимости компании. На стадии планирования сделки покупатели и продавцы начинают с расчета именно данного вида стоимости. Fair market value - это справедливая рыночная стоимость, то есть та стоимость, которую принима- 
ют государственные органы; стоимость, близкая к средней рыночной цене подобного рода объектов. Она основывается на размере компании, объеме ассортимента продукции или услуг, доступе к капиталу, уровне эффективности управления, уровне рентабельности и ликвидности активов компании, уровне заемного финансирования, рынке сбыта и клиентской базе.

- При расчете данного вида стоимости покупатели должны учитывать, что справедливая рыночная стоимость - это лишь образная стоимость, рассчитанная на основе показателей действующих на $\mathbf{0}$ рынке организаций. Соответственно, она является базовой на начальном этапе переговоров.

В отличие от справедливой рыночной стоимости, Investment value, или инвестиционная стоимость, - это стоимость данного предприятия для конкретного инвестора с его индивидуальными предшпочтениями, мотивами и целями, возможностями и ограничениями. Для каждого покупателя интестиционная стоимость (или стратегическая) будет иметь свою величину, так как каждый из них §обладает своими стратегическими преимуществами и мотивами. В связи с этим целесообразно говорить о том, что на момент переговоров покупатель и продавец должны быть осведомлены о стратегических возможностях друг друга, для того чтобы инвестиционная стоимость была справедливой для обеих сторон.

() Превышение инвестиционной стоимости над рыночной - это та максимальная стоимость, на ко乞торую покупатель рассчитывает в результате заключения сделки. А премия, выплаченная покупаотелем продавцу сверх рыночной стоимости за сделку, потенциально уменьшает прибыль, которую -может получить покупатель.

๓Для того чтобы определить ту или иную стоимость компании (будь то рыночная или инвестициононая), необходимо использовать один из методов оценки стоимости компании. Все методы оценки гстоимости компании являются рыночными, так как они учитывают обстановку на рынке на дату > расчета оценки, риски и ожидания инвесторов, а также ответную реакцию рынка на совершение с сделки купли-продажи [Ивашковская, 2009].

Но, несмотря на все преимущества данного подхода, необходимо помнить о том, что каждый подход нацелен на оценку компаний с определенными характеристиками. Так, например, компании, принадлежащие к отрасли автомобилестроения, оцениваются иначе, нежели компании в нефтегазовой отрасли.

Исходя из данного предположения, проанализируем, какой способ оценки компании наилучшим образом подходит для оценки компании нефтегазового сектора.

Нефтегазовые компании отличаются от компаний, деятельность которых происходит в других отраслях. Перечислим основные их отличительные особенности:

- непредсказуемые денежные потоки и выручка вследствие колебаний цен на нефть и газ;

- будущая выручка нефтяной компании генерируется с помощью запасов полезных ископаемых, которые составляют основную часть активов таких компаний;

- $\quad$ нефтегазовые компании характеризуются большим показателем капитальных расходов (или CapEx), так как для открытия новых месторождений требуется высокий уровень финансирования;

- деятельность нефтегазовых компаний характеризуется цикличностью, что также объясняется колебаниями цен на нефть.

Итак, представив некоторые отраслевые особенности, можно заключить, что существует несколько минусов при оценке методом дисконтированных денежных потоков (DCF) стоимости компании, принадлежащей нефтегазовой отрасли. В основе данного метода лежит дисконтирование денежных потоков, величина которых может быть уменьшена вследствие высокого показателя капитальных расходов, или СарЕх. Также сложность заключается в том, что нельзя спрогнозировать верный уровень роста компании из-за сложности предсказания эффективности ее деятельности.

Далее необходимо уделить внимание мультипликативному, или, как его иначе называют, сравнительному подходу. В его основе лежит утверждение о том, что покупатель не станет переплачивать за компанию больше суммы, которая характерна для компании-аналога на том же рынке. Основное 
преимущество данного подхода заключается в том, что стоимость компании, оцененная с его помощью, является наиболее справедливой, ведь она образуется, исходя из информации о биржевых ценах на акцию компаний-аналогов, а также на оценочную стоимость компании-аналога.

Ч Что касается оценки стоимости компании с помощью мультипликатора, то для промышленного ○-производства наилучшим образом подходит EV/EBITDA (стоимость компании/прибыль до уплаты амортизации, налогов и \%). Также данный мультипликатор подходит при оценке капиталоемких

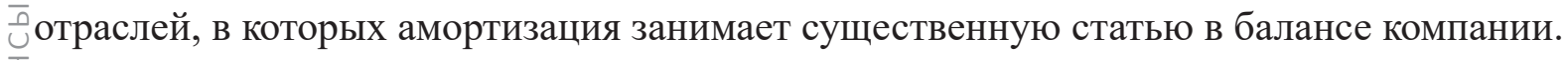

Процесс оценки стоимости компании происходит с использованием, как правило, среднеотрасле$\underset{\ominus}{\searrow}$ вого показателя. Он вычисляется как среднее значение мультипликатора EV/EBITDA нескольких шкомпаний-аналогов, которые имеют общие характеристики в размере, в величине выручки, прибытли, количестве работников и прочих показателях.

Показатель EBITDA лучше остальных показывает способность компании обслуживать долг, ею привлеченный.

В соответствии с определениями стоимости премия за контроль, или control premium, при заключении сделки M\&A - это разница между справедливой рыночной и стратегической стоимостями. (0)Но, как утверждают Эванс и Бишоп, название этой премии отнюдь не передает весь ее смысл, хотя เn ной премии состоит в достижении синергетического эффекта по итогам сделки. Соответственно, бв данной статье премия, уплаченная при заключении сделки M\&A, будет называться премией по-

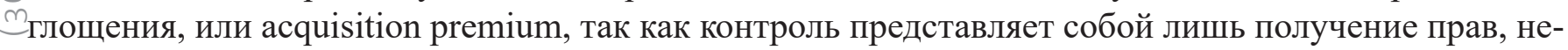
ојобходимых для активизации полученных синергетических эффектов.

Формула, по которой может быть рассчитана acquisition premium, представлена далее:

$A P R=\frac{P-F M V}{F M V} \times 100 \%$,

при этом $A P R>0, P=f(I V B, x 1, \ldots x n)$,

где $A P R \equiv$ премия за сделку, уплаченная компанией-продавцом компании-мишени;

$\mathrm{P} \equiv$ цена, уплаченная инвестором за приобретаемую компанию;

$F M V \equiv$ справедливая рыночная стоимости компании - мишени;

$I V B \equiv$ инвестиционная стоимость компании-мишени для определенного покупателя с определенными мотивами;

$x 1 \ldots x n \equiv$ факторы, которые могут оказывать влияние на величину стоимости сделки в зависимости от характера сделки.

Помимо того условия, что премия за сделку, или $A P R$, должна быть величиной положительной, она также должна быть меньше прибыли от сделки, иначе сделка считается невыгодной для компаниипокупателя. Иначе говоря, опираясь на формулу (2), $[V A B-(V A+V B)]>A P R$.

Для определения оптимальной премии в сделках M\&A в нефтегазовом секторе для начала необходимо выявить факторы, оказывающие влияние на формирование премии для любых сделок. Исследования по данному вопросу выделяют следующие факторы:

- финансовые показатели компании-мишени;

- финансовая синергия;

- операционная синергия;

- выбор способа оплаты сделки;

- синергия от устранения неэффективного управленческого аппарата компании-мишени;

- синергия от диверсификации активов;

- синергетические эффекты от приобретения стратегических преимуществ;

- наличие/отсутствие конкурентов на этапе поглощения компании-мишени. 
Далее проанализируем, как специалисты в данной области рассматривали вышеуказанные факторы в своих работах.

1. Финансовые показатели компании-цели. Даже в теории очевидным становится тот факт, что детерминант финансовых показателей компании-мишени влияет на формирование рыночной стоимости компании-цели, а соответственно, и на величину премии. Но по данному вопросу существует ряд исследований, говорящих о влиянии данного детерминанта на премию. Так, Волклинг и Эдмистер [Walkling, Edmister, 1985] в 1985 году выявили зависимость между формированием величины премии и рядом финансовых показателей, а именно: величиной чистого оборонного капитала компании и соотношением между рыночной и балансовой стоимостью активов компании, отношением долга к активам; а также типом слияния, наличие конкурентов приобретения компании-цели в период сделки, величины приобретаемого пакета акций. А немного позже, в 1987 году, Роуз [Rose, 1987] доказал, что доходность собственного капитала компании-мишени статистически значима для формирования премии.

2. Финансовая синергия. Эффекты от финансовой синергии выражаются в следующих выгодах для компаний: уменьшение налоговых обременений, снижение средневзвешенной стоимости привлечения капитала, а также увеличение долговой нагрузки.Сласки и Кейвз [Slusky, Caves, 1991] нашли положительную зависимость между премией и эффектами от финансовой синергии, которые выражаются в уменьшении налогового обременения и уменьшении средневзвешенной стоимости привлечения капитала. Долговая нагрузка компании-цели имеет отрицательную зависимость от премии, согласно исследовательской работе Волклига и Эдмистера [Walkling, Edmister, 1985].

3. Операционная синергия. Опираясь на статистические данные, можно сделать вывод о том, что обычно выгоды от операционной синергии завышены со стороны компании-покупателя, соответственно, часто покупатель переплачивает за операционную синергию. Также, как правило, величина синергетических эффектов положительно коррелирует с показателем acquisition premium. К такому выводу приходят такие зарубежные авторы, как Сировер, Гупта и Герчак, Сласки и Кейз [Sirower, 1997; Gupta and Gerchak, 2002; Slusky, Caves, 1991]. Операционная синергия достигается в основном за счет вертикальной интеграции. Следствия операционной синергии: объединение производственных, инновационных и научных технологий, оптимизация использования ресурсов и перевозок, расширение ассортимента продукции, выход на новые рынки сбыта производимой продукции и услуг.

4. Выбор способа оплаты сделки. Согласно тому факту, что величина налоговых отчислений зависит от способа финансирования сделки, которым она должна быть оплачена, можно говорить о выявленной зависимости между премией и способом оплаты сделки, а именно: премия имеет более высокое значение при оплате денежными средствами, нежели акциями. К такому выводу пришли Лейн, Янг, Уонсли, исследовав 203 сделки в период 1970-1978 гг. [Wansley, Lane and Yang, 1983].

5. Синергия от устранения неэффективного управленческого аппарата компании-мишени. В 1965 г. Манне впервые исследовал вопрос о том, что при более правильном и корректном способе управления компанией образуются синергетические выгоды. А в 1983 г. Малатеста обнаружил зависимость между премией и синергетическим эффектом от эффективного управленческого аппарата [Malatesta, 1983; Manne, 1965].

6. Синергия от диверсификации активов. Данный фактор рассматривается с точки зрения типа интеграции. Элгерс и Кларк [Elgers and Clark, 1980] выявили следующую зависимость между типом интеграции и премией: при конгломератном слиянии премия значительно выше, чем при вертикальном или горизонтальном. Данный факт объясняется тем, что при конгломератном типе слияния чаще всего происходят вражеские поглощения, а получить свое и сломить сопротивление со стороны топ-менеджента компании-цели можно, заплатив высокую премию за сделку акционерам компании. Этим фактом также объясняется меньшая, по сравнению с остальными видами интеграции, прибыльность конгломератных поглощений.

7. Синергетические эффекты от приобретения стратегических преимуществ. Исследования по данному вопросу, проведенные Смитом и Тригеоргисом [Smith, Triantis, 2004], показали, 
что компания-покупатель извлекает из сделки не только операционные и финансовые выгоды, но и стратегические эффекты. Данная стратегическая возможность тоже имеет свое стоимостное выражение. Но точная статистическая зависимость между величиной премии и стратегическими синергетическими эффектами не была выделена ни в одном из существующих на данный момент исследований. Это объясняется отсутствием соответствующих данных. Но при построении регрессионной модели авторы сталкиваются с тем, что полученная модель объясняет только около $60 \%$ результата премии, а остальные $40 \%$ остаются в необъясненной части премии. Именно выгоды от полученных стратегических преимуществ принято относить к этим $40 \%$.

8. Наличие или отсутствие конкурентов на этапе поглощения компании-мишени. Данный фактор, несомненно, оказывает воздействие на величину выплачиваемой премии. Эмпирические исследования таких авторов, как Вэрайа, Сласки и Кейвз (исследовали 100 компаний в реальном секторе экономики) [Varaiya, 1987], а также Волклинг и Эдмистер [Walkling, Edmister, 1985] подтвердили это предположение и обнаружили положительную связь между величиной премии и наличием конкурентов на приобретение одной и той же компании. Данная зависимость факторов объясняется желанием двух конкурентов приобрести актив, вследствие чего показатель премии будет повышаться, пока продавца не удовлетворит цена, а в выигрыше не останется один из конкурентов. Исходя из этого, можно говорить о том, что премия не редко бывает завышенной в стремлении соперников выиграть в конкурентной борьбе за желаемый актив.

Данные факторы так или иначе оказывают либо положительное, либо отрицательное влияние на образование величины премии, по мнению предыдущих исследователей.

Переходя непосредственно к определению премии в сделках слияния и поглощения в нефтегазовом секторе, необходимо отметить, что способом достижения данной цели является построение автор드응 алгоритма, а также построение универсальной эконометрической многофакторной регрессионной модели.

Начнем с построения эконометрической многофакторной модели. В данной статье проводится исследование количественных и качественных данных, касающихся сделок M\&A. В исследовании принимают участие сделки на рынке слияний и поглощений в нефтегазовой отрасли в период с 2009 по 2014 г. Основной источник сбора данных - международная база сделок слияний и поглощений Bureau van Dijk Zephyr [Zephyr Annual M\&A Report Global; Zephyr Week in Review].

Первоначальный отбор сделок M\&A будет отвечать следующим основным требованиям: сделки слияния и поглощения, проводимые в нефтегазовом секторе (в мировом измерении); сделки, объявленные закрытыми в промежуток с 2009 по 2014 г; сделки, относящиеся к различным ценовым категориям.

Первоначальное формирование генеральной совокупности сделок М\&А (сбор и обработка информации и данных по сделкам M\&A) проводилось по следующим требованиям и критериям:

1. Тип сделки: поглощении, слияние (deal type: Acquisition, Merger).

2. Метод оплаты: наличные, акции (methods of payment: Cash, Shares).

3. Текущий статус сделки: подтверждено завершение сделки (current deal status - completed confirmed).

4. Период времени, в котором сделка была произведена: 01.01.2010-31.12.2014 (time period: on and after 01.01.2010 and up to and including 31.12.2014).

5. Отрасль, к которой относились компании, участвующие в сделке: так как в базе Zephyr в классификации компаний отсутствует дословное название «нефтегазовая отрасль», было решено выбрать компании, как-либо относящиеся к добыче, переработке или транспортировке нефти, газа и прочих смежных продукций (oil and gas extraction, manufacture coke and refined petroleum products, chemicals e.t.c.).

Так, основываясь на данной классификации, была получена генеральная выборка, состоящая из 4519 сделок, которые были завершены в течение последних пяти лет в нефтегазовом секторе. 
Но такого количества качественных данных достаточно мало для проведения достоверного исследования и получения надежных результатов. Поэтому было решено ввести в отбор еще и финансовые показатели компании-мишени (то есть количественные данные). Финансовые показатели компании-покупателя не будут рассмотрены в данном исследовании, так как оно ориентировано на 亡получение оценки оптимальности премии, уплачиваемой покупателем продавцу, которая во мно^гом зависит от уровня финансовой составляющей компании-продавца.

Далее было решено сделать отбор полученной генеральной совокупности из 4519 сделок, добавив следующие финансовые показатели компании-мишени и сделки в общем, которые, по предположительному мнению авторов, могли бы повлиять на величину уплачиваемой премии:

6. Конечная стоимость сделки (deal value th USD).

7. Процент приобретенных компанией-покупателем акций по условиям совершения сделки (acquired stake $(\%)$ ).

8. 3. Предлагаемая цена за акцию (offer price USD).

9. Рыночная капитализации компании-мишени за последний год (Pre-deal target market capitalisation (Last available year) th USD).

10. 5. Цена одной акции компании-мишени за 3 месяца до объявления о сделке (Target stock price 3 months prior to announcement USD).

11. 6. Показатель отношения операционной выручки на оборачиваемость компании-мишени (Target operating revenue/turnover th USD Last avail. yr).

12. ЕВIT компании-мишени (Target EBIT th USD Last avail. yr).

13. Прибыль компании-мишени до налогообложения (Target profit before tax th USD Last avail. yr).

14. Прибыль компании-мишени после налогообложения (Target profit after tax th USD Last avail. yr).

15. Совокупные активы компании-мишени (Target total assets th USD Last avail. yr).

16. 11. Показатель собственного капитала компании-мишени (Target shareholders funds th USD Last avail. yr).

17. 1Количество размещенных акций компании-мишени (Target number of outstanding shares First avail. yr).

Некоторые из вышеперечисленных показателей не будут рассмотрены в чистом виде, то есть они будут применены в расчетах.

Далее авторами было выдвинуто предположение, что такие фиктивные переменные, как трансграничный тип сделки, вид интеграции сделки (горизонтальная, вертикальная или конгломерат), враждебное отношение компании-покупателя к сделке, наличие информационной асимметрии, метод финансирования сделки оказывают непосредственное влияние на формирование премии.

Регрессионный анализ проводился в статистическом пакете STATA.

Для статистического пакета STATA данные для этих переменных были обозначены следующим образом.

Таблица 1

Описание фиктивных переменных

\begin{tabular}{|c|c|c|}
\hline Вид классификации & \multicolumn{2}{|c|}{ Условия для Stata } \\
\hline Трансграничный тип сделки & Если из одной страны - 0 & Если из разных - 1 \\
\hline Способ финансирования сделки & Если Shares - 1 & Если Cash и остальное - 0 \\
\hline Наличие информационной асимметрии & $\begin{array}{c}\text { Отсутствие информационной } \\
\text { асимметрии - } 0\end{array}$ & $\begin{array}{c}\text { Существует факт присутствия } \\
\text { информационной асимметрии - } 1\end{array}$ \\
\hline
\end{tabular}




\begin{tabular}{|c|c|c|}
\hline $\begin{array}{c}\text { Враждебное отношение компании-покупателя к } \\
\text { сделке }\end{array}$ & Если дружественное -0 & Если враждебное -1 \\
\hline Вид интеграции* (А: вертикальный тип \\
$\begin{array}{c}\text { интеграции) } \\
\text { (В: вид интеграции - конгломерат) }\end{array}$ & $0-$ не вертикальный тип & $1-$ вертикальный тип \\
\hline
\end{tabular}

*ㅜㄴ интеграции имеет три значения, поэтому для его моделирования используются две фиктивные переменные: вер-тикальный тип и конгломерат. Горизонтальный тип интеграции однозначно определяется равенством нулю этих двух переменных.

Обозначения и расчеты некоторых указанным выше переменным были даны с учетом существющего научного и эмпирического опыта исследовательских работ. Например, наличие информашционнй асимметрии предполагает, что один из участников сделки не владеет всей информацией о другой компании, что необходимо для планирования сделки, а также для успешного ее заверше$\checkmark$ ния. База Zephyr не содержит такой информации, именно поэтому было решено, что когда компания-покупатель приобретает сразу 100\% активов компании-мишени, то присутствует информационная асимметрия; а когда покупатель увеличивает свою долю владения активами продавца, то считалось, что в таком случае асимметрия отсутствует. Что касается враждебного или дружеского отношения к сделке компании-покупателя, то враждебным оно считалось в случае покупки сразу $100 \%$ акций наличными средствами, а также когда компания-покупатель делает тендерное предоложение компании-цели. Тендерное предложение может характеризовать и дружескую сделку в случае, когда такое предложение делают менеджменту компании-цели, но так как база Zephyr не содержит информации данного рода, то делаем допущение о том, что тендерное предложение было сделано акционерам компании.

Таким образом, после определения необходимых для исследования критериев отбора сделок, вся необходимая информация о которых представлена в базе Zephyr, из ранее сформированной генеральной совокупности авторы получили 141 сделку.

Зависимой переменной в эконометрической регрессионной модели является величина премии, уплачиваемая покупателем продавцу, или acquisition premium. Необходимо оговориться, что в STATA эта зависимая переменная обозначается как «ACQ_PREM». Премия может выражать более высокую цену за акцию на момент сделки, чем ранее торгуемая цена за эту же акцию. Премия есть разница между предложенной ценой и ценой до предложения в процентном выражении. Иными словами, премия - показатель того, переплатил ли покупатель (или продавец невыгодно продал свои активы) или же сделка прошла успешно.

При отборе данных в базе Zephyr был обнаружен показатель Bid Premium, который является той самой премией. Но существует необходимость пересчитать величину премии для каждой сделки, так как в базе Zephyr премия рассчитывается следующим образом:

Bid Premium $=(Y-X) / X^{*} 100 \%$,

где $Y \equiv$ предлагаемая цена за акцию, $X \equiv$ цена за акцию в день до объявления о сделке.

Но в данной статье принимается теория, предложенная Нильсеном и Мелихером [Nielsen, Melicher, 1973], о том, что в расчете премии надежнее всего используется показатель цены акции за 3 месяца до объявления о сделке. Данные о котировках акций берутся за 3 месяца до объявления о сделке M\&A, для того чтобы избежать необъективности в цене котировок из-за слухов и инсайдерской информации.

Итак, величина уплачиваемой acquisition premium пересчитана в данной работе по следующей формуле:

Acquisition premium $=(Y / X-1) * 100 \%=(Y-X) / X * 100 \%$,

где $Y \equiv$ предложенная цена за акцию уже после объявления о сделке (Offer price USD), $X \equiv$ цена акции компании-мишени за 3 месяца до объявления о сделке (Target stock price 3 months prior to announcement USD).

При пересчете на первый взгляд обнаружено некоторое различие в показателях премии (в своем большинстве премия, указанная в общем доступе, оказывается завышенной). 
Далее необходимо проверить зависимую переменную ACQ_PREM на нормальность распределения, для того чтобы на выходе получить эффективную и несмещенную оценку коэффициентов.

Для данной цели был построен график для оценки плотности распределения:

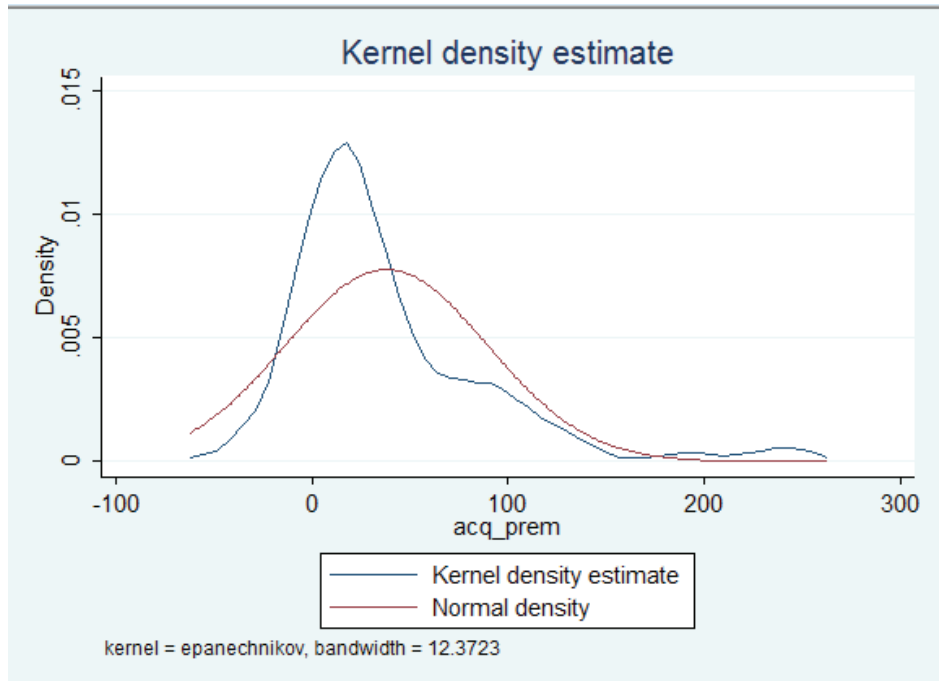

\begin{tabular}{|l|l|}
\hline \multicolumn{1}{|c|}{$\begin{array}{c}\text { Обозначения на } \\
\text { английском языке }\end{array}$} & \multicolumn{1}{|c|}{$\begin{array}{c}\text { Перевод обозначений на русккий } \\
\text { язык }\end{array}$} \\
\hline Kernel density estimate & Оценка плотности распределения \\
\hline Normal density & $\begin{array}{l}\text { Функция плотности нормального } \\
\text { распределения }\end{array}$ \\
\hline
\end{tabular}

График 1. Плотность распределения переменной $A C Q \_P R E M$

На графике 1 приведены кривые плотности распределения зависимой переменной ACQ_PREM и плотности нормального распределения. Как видно, кривая плотности нашей переменной характеризуется более высоким эксцессом (крутовершинностью) по сравнению с нормальным распределением и правой асимметрией.

Чтобы убедиться в том, что распределение независимой переменной ACQ_PREM отличается от нормального, необходимо провести Тест Шапиро-Франсиа.

Таблица 2

Тест Шапиро-Франсиа на нормальность распределения зависимой переменной ACQ_PREM Shapiro-Francia W' teat for normal data

\begin{tabular}{|c|c|c|c|c|c|}
\hline Variable & Obs & W' & V' & z & Prob $>$ z \\
\hline acq prem & 141 & 0,83634 & 19,796 & 6,034 & 0,00001 \\
\hline
\end{tabular}

Итак, Тест Шапиро-Франсиа выявил отличие от нормального распределения (Р < уровня значимости 0,05).

Следующий шаг - анализ выбросов. Для того чтобы привести ACQ_PREM к нормальному распределению, необходимо избавиться от выбросов. Для достижения этой цели строим график выбросов BoxPlot для зависимой переменной.

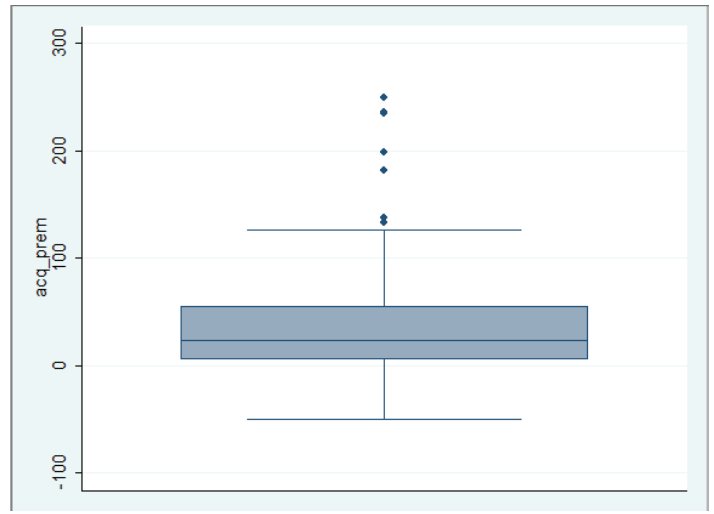

График 2. BoxPlot для зависимой переменной $A C Q \_P R E M$ 
Итак, все значения премии больше 130\% были удалены. Далее потребовалось дважды строить BoxPlot, a соответственно, дважды удалять выбросы. В первом случае удалили сделки с премией больше $110 \%$, а во втором - с премией больше $100 \%$ и дисконтом меньше 50\% (дисконт - отрицательная премия).

- После трех попыток привести переменную ACQ_PREM к нормальному распределению получили следующий график распределения (табл. 3) и провели тест на нормальность распределения (граüфик 3).

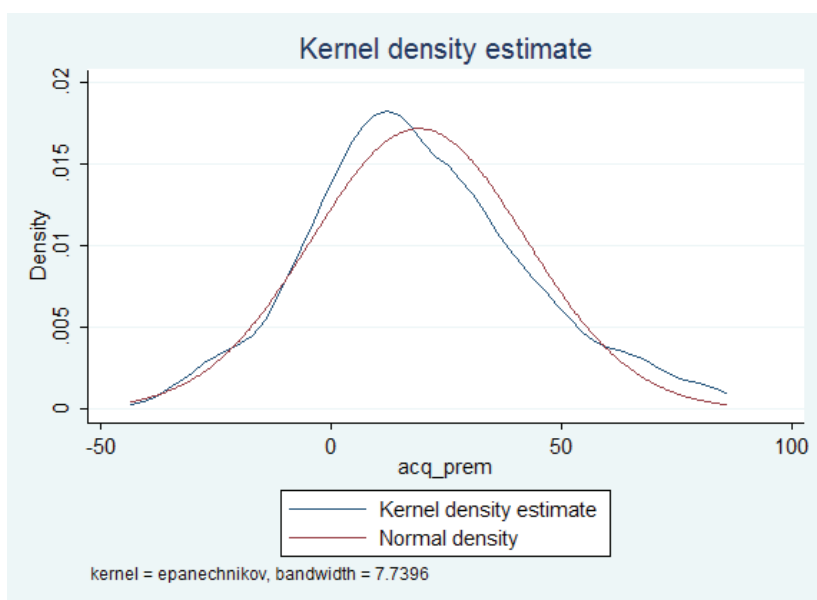

\begin{tabular}{|l|l|}
\hline \multicolumn{1}{|c|}{$\begin{array}{c}\text { Обозначения на } \\
\text { английском языке }\end{array}$} & \multicolumn{1}{|c|}{$\begin{array}{c}\text { Перевод обозначений на русккий } \\
\text { язык }\end{array}$} \\
\hline Kernel density estimate & Оценка плотности распределения \\
\hline Normal density & $\begin{array}{l}\text { Функция плотности нормального } \\
\text { распределения }\end{array}$ \\
\hline
\end{tabular}

График 3. Распределение переменной ACQ_PREM после удаления выбросов

Таблица 3

Тест Шапиро-Франсиа на нормальность распределения зависимой переменной ACQ_PREM после удаления выбросов Shapiro-Francia $\mathrm{W}^{\prime}$, teat for normal data

\begin{tabular}{|c|c|c|c|c|c|}
\hline Variable & Obs & W' & V' & z & Prob > z \\
\hline acq prem & 113 & 0,98545 & 1,464 & 0,760 & 0,22363 \\
\hline
\end{tabular}

Согласно графику 3 и таблице 3, после удаления выбросов получили распределение, близкое к нормальному. Тест Shapiro-Francia показал, что различия между плотностью распределения изучаемой переменной и плотностью нормального распределения не являются статистически значимыми (вероятность $P$-значения, равная $0.22>\alpha$, где $\alpha$ - уровень значимости). Считаем распределение зависимой переменной ACQ_PREM нормальным. Количество сделок после удаления выбросов равно 113.

Рассчитаем основные статистические характеристики случайной величины ACQ_PREM:

Таблица 4

Основные статистические характеристики ACQ_PREM

\begin{tabular}{|c|c|c|c|c|c|}
\hline Variable & Obs & Mean & Std. Dev. & Min & Max \\
\hline acq prem & 113 & 19,13891 & 23,32084 & $-35,5636$ & 78,30969 \\
\hline
\end{tabular}

Итак, выборка из 113 наблюдений за последние пять лет показала, что среднее значение премии составляло 19,139\%, что хорошо согласуется с результатами других исследований; стандартное $\sqcup$ отклонение $=23,3$, что характеризует разброс значений премии относительно среднего значения (мат. ожидания). Максимум выплаченной премии среди сделок в выборке $-78,3 \%$, минимальное - значение равно $-35,6 \%$.

Следующий этап исследования - это анализ независимых количественных переменных. Выяснилось, что распределения всех количественных переменных отличаются от нормального, однако 
удалять наблюдения авторы не считают целесообразным, поскольку это сильно уменьшит объем выборки. Отметим, однако, что значительных отклонений не наблюдается.

Далее приведем обозначения этих независимых количественных переменных для STATA, а также фиктивных переменных, которые введены в модель 4.

Таблича 5

Обозначение количественных переменных

\begin{tabular}{|c|c|}
\hline Обозначение в Stata & Расшифровка переменной \\
\hline deal_val & Конечная стоимость сделки (deal value th USD) \\
\hline in_stake & Доля акций компании-мишени, которой уже владеет компания-покупатель на момент сделки (initial stake, \%) \\
\hline acq_stake & Процент приобретенных акций компанией-покупателем по условиям совершения сделки (acquired stake, \%) \\
\hline mark_cap & Рыночная капитализация компании-мишени за последний год (Pre-deal target market capitalization (Last available \\
уеar) th USD)
\end{tabular}

Таблица 6

Описание фиктивных переменных

\begin{tabular}{|c|c|}
\hline Обозначение в STATA & Расшифровка переменной \\
\hline transnat & Трансграничный тип сделки \\
\hline рау_meth & Способ финансирования сделки \\
\hline inf_as & Наличие информационной асимметрии \\
\hline hostile & Враждебное отношение компании-покупателя к сделке \\
\hline vert_in/congl_int & Вид интеграции* \\
& (А: вертикальный тип интеграции) \\
& (В: тип интеграции - конгломерат) \\
\hline
\end{tabular}

Следующий этап включает в себя корреляционный анализ. Проведем отбор количественных переменных, по которым будет проведен регрессионный анализ. Должны выполняться следующие требования: переменные должны оказывать влияние на размер премии, должна отсутствовать мультиколлинеарность между факторами (чтобы факторы никак не влияли друг на друга). Очень слабую корреляцию с премией имеет переменная in_stake, то есть доля акций, которыми владеет покупатель до совершения сделки, никак не влияет на величину премии, согласно нашему исследованию. Факторы ebit и profit before tax сильно коррелируют с profit after tax. Соответственно, два вышеназванных фактора тоже необходимо удалить. Также очевидно, что sh_funds и tot_assets должны быть удалены, так как они сильно взаимосвязаны с остальными переменными и могут быть найдены через них. В итоге регрессионный анализ показал, что величина премии зависит от шести количественных факторов:

Корреляционная матрица зависимости величины премии от количественных переменных

acq premacq st $\sim$ mark cap turnover prof a $\mathrm{t}$ out sh $\sim \mathrm{s}$ deal val

acq prem 1,000

\begin{tabular}{l|r|r}
\hline acq $\_$st $\sim$ e & 0,062 & 1,000
\end{tabular}

mark_cap $\quad 0,178 \quad 0,074$

\begin{tabular}{l|r|r|r|} 
turnover & $-0,067$ & 0,061 & 0,382 \\
\hline
\end{tabular}

prof_a_t

$0,063 \quad-0,017$

0,571

out_sh $\sim$ s

$-0,013 \quad-0,013$

$-0,110$

1,000

deal_val

$0,157 \quad 0,353$

$0,684 \quad 0,271$

1,000

0,283

\begin{tabular}{l}
$0,153 \quad 1,000$ \\
\hline
\end{tabular} 
После определения количественных факторов, оказывающих достаточно сильное влияние на величину премии, построили регрессию только с количественными переменными.

Регрессионная модель, состоящая из количественных переменных (модель 1):

$\underset{\neg}{\llcorner}$ acq prem $=\beta 0+\beta 1$ acq_stake $+\beta 2$ mark_cap $+\beta 3$ turnover $+\beta 4$ prof_a_t $+\beta 5$ out_shares $+\beta 6$ deal_ ○ेvalue.

亏алее была построена модель в статистическом пакете STATA:

Таблица 8

Результаты построения модели 1

\begin{tabular}{|c|c|c|c|c|c|c|}
\hline Source & SS & $\mathrm{df}$ & MS & & Number of obs $=$ & 113 \\
\hline Model & 5389,70174 & & 898,283624 & & $F(6,106)=$ & 1,71 \\
\hline Residual & 55522,8199 & 106 & 523,800187 & & Prob $>F=$ & 0,1245 \\
\hline Total & 60912,5216 & 112 & 543,8618 & & R-squared = & 0,0885 \\
\hline & & & & & Adj R-squares = & 0,0369 \\
\hline & & & & & Root MSE = & 22,887 \\
\hline acq prem & Coef. & Std. Err. & $\mathrm{t}$ & $\mathrm{P}>|\mathrm{t}|$ & \multicolumn{2}{|c|}{ [95\% Conf. Interval] } \\
\hline acq stake & 0,0229761 & 0,063617 & 0,36 & 0,719 & $-0,1031501$ & 0,149102 \\
\hline mark_cap & $3,14 \mathrm{E}-06$ & $1,30 \mathrm{E}-06$ & 2,42 & 0,017 & 5,71E-07 & $5,71 \mathrm{E}-06$ \\
\hline turnover & $-3,45 \mathrm{E}-07$ & $2,43 \mathrm{E}-07$ & $-1,42$ & 0,159 & $-8,27 \mathrm{E}-07$ & $1,37 \mathrm{E}-07$ \\
\hline prof_a_t & $-4,22 \mathrm{E}-06$ & $1,09 \mathrm{E}-05$ & $-0,39$ & 0,700 & $-0,0000259$ & $1,74 \mathrm{E}-05$ \\
\hline out_shares & $-4,37 E-09$ & 2,32E-09 & $-1,88$ & 0,062 & -8,97E-09 & $2,29 \mathrm{E}-10$ \\
\hline deal_val & $-7,88 \mathrm{E}-07$ & $1,24 \mathrm{E}-06$ & $-0,64$ & 0,525 & $-3,24 \mathrm{E}-06$ & $1,66 \mathrm{E}-06$ \\
\hline cons & 16,286 & 4,313072 & 3,78 & 0,000 & 7,734916 & 24,83708 \\
\hline
\end{tabular}

$\stackrel{\circ}{\circ}$ качестве модели в целом можно судить по значению коэффициента детерминации $R$-squared $=$ 0,0885. Столь низкое значение говорит о том, что лишь $9 \%$ результата величины премии объясняется тестируемыми факторами. Проверка значимости регрессии в целом проводится с помощью $F$-теста Фишера: Prob $>F=0,1245$. Это значение больше уровня значимости (даже 0,1$)$, следовательно, нет оснований отвергнуть нулевую гипотезу о равенстве всех коэффициентов нулю, что говорит о незначимости всей регрессионной модели. Проверка значимости отдельных коэффициентов осуществляется с помощью $t$-теста Стьюдента: судя по полученным данным, можно говорить о том, что значимость коэффициентов при регрессорах различная. Можно сделать вывод о том, что некоторые регрессоры в принципе не оказывают влияния на величину премии.

Далее было построено еще четыре модели путем удаления или добавления количественных и (или) фиктивных характеристик формирования премии. Итоговая, 6-я модель является наиболее эффективной из всех ей предшествующих.

Модель 6 имеет следующее уравнение:

ACQ_PREM $=3,58^{*}\left(10^{\wedge}-6\right)$ mark_cap $-3,75^{*}\left(10^{\wedge}-7\right)$ turnover $-4,13^{*}\left(10^{\wedge}-9\right)$ out_shares $-7,56^{*}\left(10^{\wedge}\right.$ -7) deal_val $+6,2086$ transnat $+6,9849$ inf_as $+11,11$ vert_int $+11,38$ congl_int.

Влияние факторов на образование премии можно описать следующим образом:

- Рыночная капитализация положительно влияет на образование величины премии. Чем выше ее показатель, тем выше премия.

- Операционная прибыль компании-мишени и количество размещенных акций оказывают занижающий эффект на образование показателя премии.

- $\quad$ Характер типа интеграции положительно влияет на премию, когда компании проводят либо вертикальное, либо конгломератное слияние.

- Также исследование показало, что информационная асимметрия положительно влияет на премию. Можно предположить, что в неведении покупатель оценит стоимость компании, а соответственно и величину премии, лучше, чем, если бы он был знаком с компанией-ми- 
шенью ранее. Возможно, присутствие информационной асимметрии исключит предвзятое отношение, а также сделает его оценку объективной.

Итак, подводя итоги, необходимо сказать, что если рассматривать только количественные переменные, то объясняющая способность модели $R^{2}=18 \%$, а после введения фиктивных переменных ${ }_{-}^{-1} R^{2}$ увеличился практически в два раза. Можно сделать промежуточный вывод о том, что введение фиктивных переменных оправдано и улучшает качество прогнозирования.

Далее в исследовании было проведено тестирование итоговой модели 6: анализ остатков регрессии. Первоначальная ступень в анализе остатков регрессии - создание новой переменной. Сначала

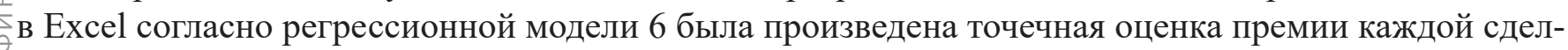
шки. Затем вычислена величина Error, как разность между рассчитанной ранее премией и точечной 品оценкой (REG). После того как Error была вычислена для каждой сделки, создали новую перемен$\stackrel{\sim}{\leq}$ ную в STATA со значениями остатков, которая была названа соответствующе - Error.

Построим гистограмму распределения остатков регрессии:

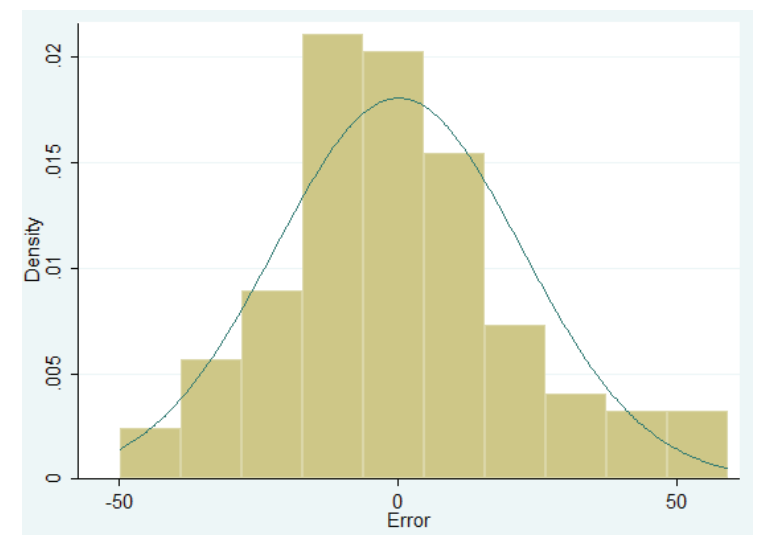

Гистограмма 1. Распределения остатков регрессии

Построили также график плотности распределения (Error):

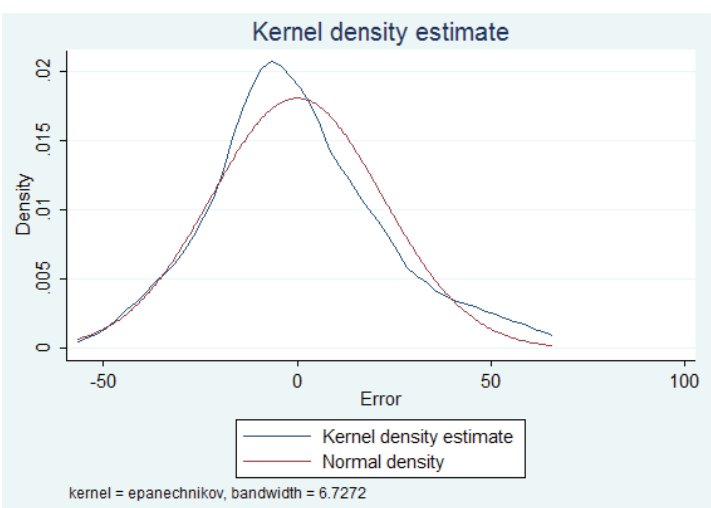

\begin{tabular}{|l|l|}
\hline \multicolumn{1}{|c|}{$\begin{array}{c}\text { Обозначения на } \\
\text { английском языке }\end{array}$} & \multicolumn{1}{|c|}{$\begin{array}{c}\text { Перевод обозначений на русккий } \\
\text { язык }\end{array}$} \\
\hline Kernel density estimate & Оценка плотности распределения \\
\hline Normal density & $\begin{array}{l}\text { Функция плотности нормального } \\
\text { распределения }\end{array}$ \\
\hline
\end{tabular}

График 4. Плотность распределения (Error)

Гистограмма показывает плавные возрастания и убывания остатков регрессии, плотность распределения близка к нормальной, хотя имеет место незначительная правосторонняя асимметрия.

Связь наблюдаемых данных к теоретическому распределению можно зрительно оценить на графике квантилей. Теоретическое распределение подходит к эмпирическим данным, если наблюдения находятся на прямой линии. 


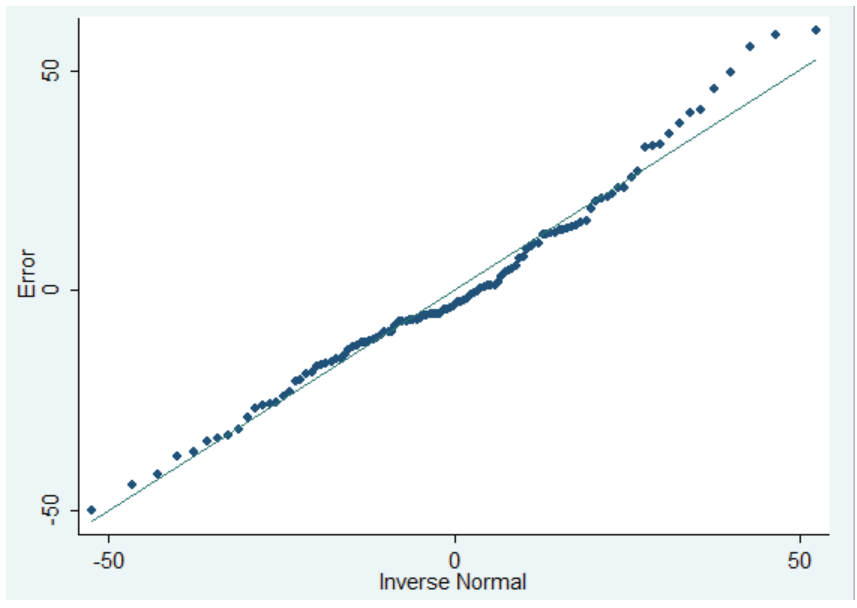

График 5. Квантиль-квантиль

Как видно на графике 5, наблюдения либо лежат на прямой, либо расположены к ней очень близко, хотя присутствуют незначимые отклонения.

Далее необходимо проверить гипотезу о нормальности распределения, проведя тесты Shapiro$\neg$ Francia, Shapiro-Wilk, Skewness/Kurtosis test.

Таблица 9

Тест Шапиро-Франсиа на нормальность распределения Error

\begin{tabular}{|l|r|c|c|c|c|}
\hline \multicolumn{5}{|c|}{ Shapiro-Francia W" test for normal data } \\
\hline Variable & Obs & W" & V" & Z & Prob > z \\
\hline Error & 113 & 0,98020 & 1,994 & 1,375 & 0,08460 \\
\hline
\end{tabular}

P-value $=0,08$, то есть больше 0,05, значит, распределение остатков регрессии можно считать нормальным.

Таблииа 10

Тест Шапиро-Уилка на нормальность распределения Error

\begin{tabular}{|l|c|c|c|c|c|}
\hline \multicolumn{5}{|c|}{ Shapiro-Wilk W test for normal data } & \\
\hline Variable & Obs & W & V & z & Prob > z \\
\hline Error & 113 & 0,97901 & 1,920 & 1,457 & 0,07225 \\
\hline
\end{tabular}

$P$-value = 0,07, то есть больще 0,05, значит, распределение остатков регрессии также можно считать нормальным.

Таблица 11

Тест Skewness/Kurtosis на нормальность распределения Error

\begin{tabular}{|l|crrr|r|}
\hline & \multicolumn{4}{|c|}{ Skewness/Kurtosis tests for Normality } & \\
\hline Variable & Obs & $\operatorname{Pr}$ (Skewness) & Pr (Kurtosis) & adj chi2 (2) & Prob > chi2 \\
\hline Error & 113 & 0,0461 & 0,432 & 4,66 & 0,0975 \\
\hline
\end{tabular}

P-value =0,097, то есть больше 0,05, значит, по итогам и этого теста можно говорить о том, что распределение остатков регрессии также можно считать нормальным.

Промежуточный вывод: нулевая гипотеза подтверждается, остатки регрессии имеют нормальное распределение, следовательно, регрессионная модель пригодна не только для того, чтобы давать точечные прогнозы, но и чтобы давать интервальные прогнозы (когда истинное значение находится в заданном интервале с заданным уровнем доверия).

Проводим тесты на гетероскедастичность. 
Необходимо проверить присутствие гетероскедастичности случайных ошибок регрессионной модели. Для этого можно провести статистический тест Бройша-Пагана. Перед проведением данного теста выдвигаем нулевую гипотезу о том, что дисперсия остатков постоянна.

Таблица 12

\section{Тест Бройша-Пагана}

\begin{tabular}{|} 
Breusch-Pagan / Cook-Weisberg test for heteroskedasticity \\
\hline H0: & Constant Variance \\
\hline Variables: & fitted values of acq prem \\
\hline & \\
\hline chi2 $(1)=$ & 0,02 \\
\hline Prob $>$ chi2 $=$ & 0,8777 \\
\hline
\end{tabular}

P-value = 0,9 превышает уровень значимости (0,05), следовательно, нет основания отвергнуть нулевую гипотезу ( $H_{0}:$ дисперсия остатков постоянна), то есть гетероскедастичность отсутствует.

Тест Уайта. Данный тест подразумевает использование остатков регрессии методом наименьших ศквадратов. В ходе теста строится дополнительная регрессия квадратов остатков на все регрессоры, попарные произведения и квадраты регрессоров.

Таблийа 13

Тест Уайта

Cameron \& Trivedi's decomposition of IM - test

\begin{tabular}{|c|c|c|c|}
\hline Sourse & chi2 & df & $\mathrm{p}$ \\
\hline Heteroskedasticity & 27,22 & 39 & 0,9223 \\
\hline Skewness & 7,96 & 8 & 0,4376 \\
\hline Kurtosis & 0,52 & 1 & 0,4714 \\
\hline Total & 35,70 & 48 & 0,9054 \\
\hline
\end{tabular}

$P$-value $=0,9054$ превышает уровень значимости $(0,05)$, следовательно, нет основания отвергнуть нулевую гипотезу ( $H_{0}:$ дисперсия остатков постоянна), то есть гетероскедастичность отсутствует.

Наличие гетероскедастичности приводит к неэффективности оценок коэффициентов регрессии методом наименьших квадратов. В нашем случае гетероскедастичность отсутствует, следовательно, оценки коэффициентов эффективны.

Проведем тест на мультиколлинеарность, оценив значение VIF для каждого из регрессоров модели.

Таблииа 14

\begin{tabular}{|l|r|l|}
\multicolumn{1}{c|}{ VIF-тест } \\
\hline \multicolumn{1}{|c|}{ Variable } & \multicolumn{1}{c|}{ VIF } & $1 /$ VIF \\
\hline mark_cap & 5,07 & 0,197418 \\
\hline deal_val & 2,82 & 0,355074 \\
\hline out_shares & 2,70 & 0,369841 \\
\hline cong_int & 1,92 & 0,520050 \\
\hline ver__int & 1,89 & 0,529269 \\
\hline turnover & 1,21 & 0,826747 \\
\hline hostile & 1,14 & 0,878002 \\
\hline transnat & 1,04 & 0,962196 \\
\hline Mean VIF & 2,22 & \\
\hline
\end{tabular}

Поскольку значения коэффициентаVIF имеют невысокие значения (а один из них на 7/100 превышает максимальный уровень), можно говорить о том, что объясняющие переменные неколлинеарны. 
Далее в исследовании была проведена оценка премии на примере сделки M\&A в нефтегазовом секторе.

Для начала был составлен ряд критериев, по которым создавалась совокупность сделок:

1. Тип сделки: слияние и поглощение.

2. Статус сделки: окончена.

3. Классификация отрасли: ТЭК (топливно-энергетический комплекс) => нефтегазовая отрасль.

4. Географическая принадлежность: любая.

${ }^{\ominus}$ Итак, для верной оценки премии в сделках слияния и поглощения необходимо определить алгошитм, по которому будет определяться верная величина премии. Ознакомившись со многими исм следованиями и руководствами по данному вопросу, авторы пришли к выводу о том, что расчет оптимальной премии в сделках M\&A в нефтегазовом секторе рекомендуется проводить согласно следующему алгоритму:

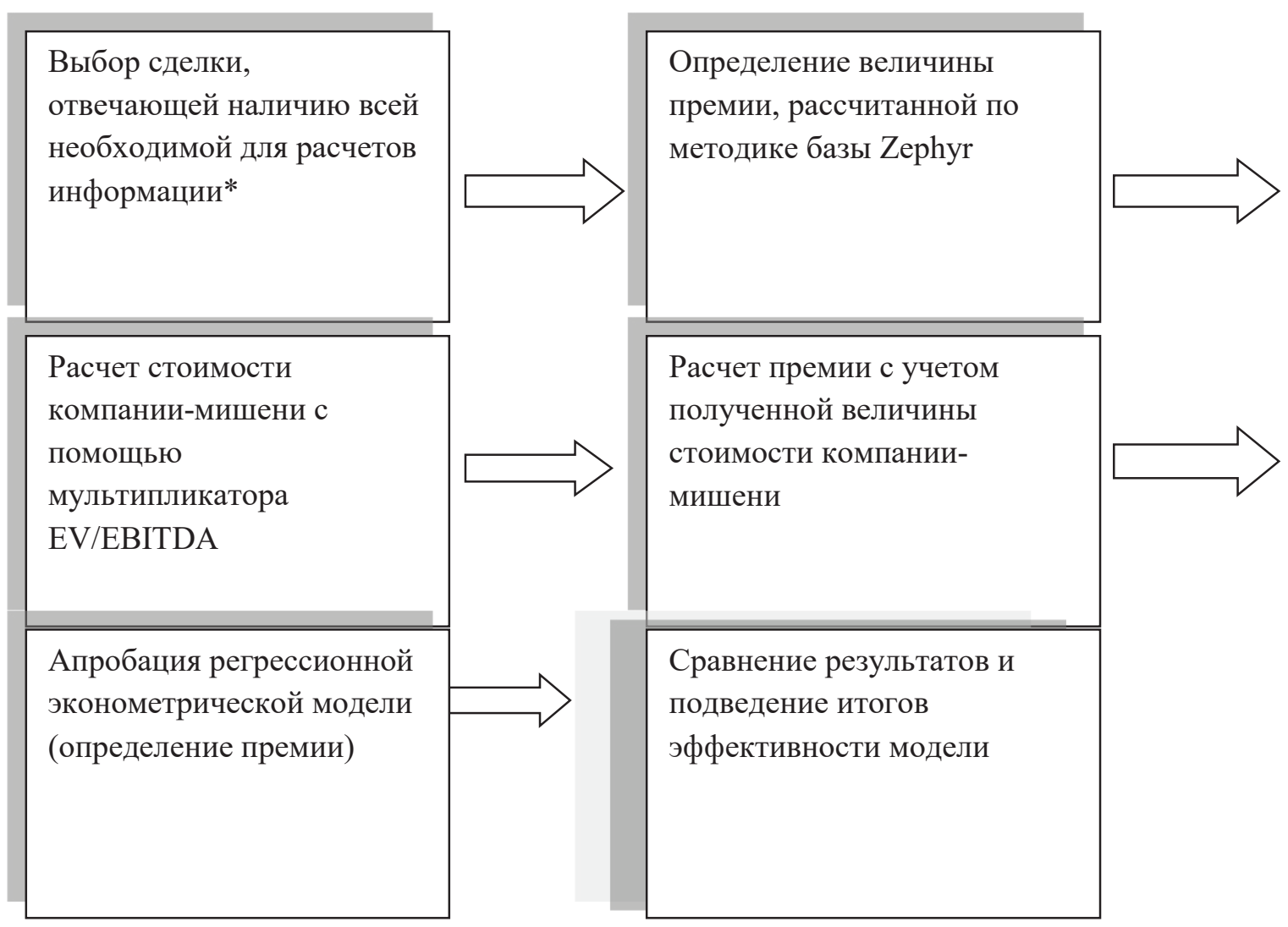

* При отборе выборки из генеральной совокупности необходимо, чтобы были известны следующие данные: компанияпокупатель; компания-цель; размер приобретаемого пакета; цена приобретаемого пакета; размер доли компании-покупателя в компании-цели после завершения сделки; общее количество акций компании-цели; находящееся в обращении; стоимость одной акции на бирже; EBITDA; классификация компаний по отраслям; страны, деятельность в которых осуществляют компании; стоимость сделки; рыночная капитализация компании-цели.

\section{Схема 1. Алгоритм расчета премии в сделках слияния и поглощения}

Для апробации модели необходимо сравнить следующие показатели acquicition premium:

1. Показатель, указанный в информации о сделке в базе Zephyr (как правило, указывают показатель, который был анонсирован на первых этапах сделки.

2. Показатель премии, который будет вычислен согласно алгоритму, указанному выше.

3. Показатель, полученный с применением эконометрической регрессионной факторной модели.

Первый этап включает выбор сделки: для расчета премии была выбрана сделка по поглощению норвежской компании REC SOLAR ASA, занимающейся утилизацией промышленных отхо- 
дов, оборудованием и производственной техникой, гонконгской компанией BLUESTAR ELKEM INVESTMENT CO LTD, которая специализируется в области нефтяной и химической промышленности. Дата анонсирования сделки: 30.05.2014; дата завершения сделки: 13.05.2015. Сделка характеризуется как вертикально интегрированная; компании-участники относятся к разным странам. ${ }_{-}$Компания-покупатель поглощает $100 \%$ акций компании-мишени.

Второй этап: расчет премии с учетом цены акции за 1 день до объявления о сделке (методика ин气 формационной базы Zephyr).

Премия рассчитывается по следующей формуле:

Acquisition premium $=(Y / X-1) * 100 \%=(Y-X) / X * 100 \%)$

殅 где $Y \equiv$ предложенная цена за акцию уже после объявления о сделке (Offer price USD), $X \equiv$ цена макции компании-мишени за 1 день до объявления о сделке.

Acquisition premium $=14,88 \%$.

Проанализировав показатель, можно сделать предположение о том, что покупатель переплатил за сделку около $15 \%$, что может быть вызвано влиянием на цену акций компании-мишени инсайдер(ㄷ) ской информации и слухов.

־ㄱ Третий этап: расчет стоимости компании-мишени с помощью мультипликатора EV/EBITDA.

Необходимые данные для расчета представлены в таблице 15.

(1)

Таблица 15

Данные для расчета стоимости компании-мишени [на основе данных Zephyr]

\begin{tabular}{|c|c|c|c|}
\hline Показатель & $\begin{array}{c}\text { EV или стоимость компании- } \\
\text { цели на момент сделки (Target } \\
\text { enterprise value USD Last. avail. } \\
\text { year) }\end{array}$ & $\begin{array}{c}\text { EBITDA компании-цели } \\
\text { (Target EBITDA USD Last } \\
\text { avail. year) }\end{array}$ & $\begin{array}{c}\text { Мультипликатор EV/ } \\
\text { EBITDA }\end{array}$ \\
\hline Величина показателя & 485331574 & 97100000 & 5 \\
\hline
\end{tabular}

Итак, мультипликатор EV/EBITDA для компании-мишени равен 4,07. Однако для расчета стоимости компании сравнительным подходом необходимо среднее значение мультипликатора по отрасли.

Таблица 16

Среднеотраслевой мультипликатор EV/EBITDA [сайт Дамодарана]

\begin{tabular}{|c|c|c|}
\hline Название отрасли & $\begin{array}{c}\text { Количество анализируемых } \\
\text { компаний }\end{array}$ & $\begin{array}{c}\text { Средний показатель } \\
\text { мультипликатора EV/ } \\
\text { EBITDA }\end{array}$ \\
\hline $\begin{array}{c}\text { Oil/Gas (Production and } \\
\text { Exploration) }\end{array}$ & 392 & 5,09 \\
\hline
\end{tabular}

Обычно, рассчитывая стоимость компании сравнительным подходом методом компаний-аналогов

с помощью нескольких мультипликаторов, при вычислении принимают вес каждого из них. Но так как в данной работе рассматривается только один мультипликатор, то он учитывается в расчетах с весом 1.

Справедливая рыночная стоимость компании-мишени, рассчитанная методом компаний-аналогов, равна:

$F M V_{\text {к-м }}=97100000 * 5.09 * 1=494239000$ долл. США.

Четвертый этап: оценка величины премии по следующей формуле:

$A P R=\frac{P-F M V}{F M V} \times 100 \%$,

где $A P R \equiv$ премия за сделку, уплаченная компанией-продавцом компании-мишени;

$\mathrm{P} \equiv$ цена, уплаченная инвестором за приобретаемую компанию;

$F M V \equiv$ справедливая рыночная стоимости компании-мишени. 
Данные для расчета премии

\begin{tabular}{|c|c|c|}
\hline Показатель & $\begin{array}{c}\text { Р (цена сделки - deal value } \\
\text { USD) }\end{array}$ & FMV (USD) \\
\hline Величина показателя & 579741085 & 494239000 \\
\hline
\end{tabular}

-Разница в показателях премий, рассчитанных различным образом, может объясняться в разном подходе к оценке стоимости компании-мишени. При этом разницу можно считать незначимой.

Гятый этап: расчет премии с использованием регрессионной модели, то есть апробация модели.

Модель выглядит следующим образом:

$A C Q$ PREM $=3,58^{*}\left(10^{\wedge}-6\right)$ mark_cap $-3,75^{*}\left(10^{\wedge}-7\right)$ turnover $-4,13^{*}\left(10^{\wedge}-9\right)$ out_shares $-7,56^{*}\left(10^{\wedge}\right.$ - 7) deal_val + 6,2086 transnat + 6,9849inf_as $+11,11$ vert_int $+11,38$ congl_int

$A C Q$ PREM=11,5\%.

Шестой этап (сравнение полученных показателей): анализируя полученные показатели, можно сделать вывод: как и ожидалось, довольно низкое значение коэффициента детерминации $R^{2}$ означает, что данное уравнение лишь на 40\% (округляя значение) объясняет общую дисперсию образования показателя премии. Несмотря на среднюю объясняющую способность итоговой модели, величина премии, полученная с помощью регрессии, довольно близко расположена к рассчитанным иными б способами значениям премии: 14,88\%, 17,3\%, 11,5\%. Можно предположить, что факторы, которые m имеют высокое влияние на формирование премии, являются неофициальными или ранее не расо сматривались.

๖Взможные пути дальнейшего усовершенствования модели: введение дополнительных перемен느의, которые могут быть известны путем более подробного и глубокого анализа сделок, а также п мотивов и целей.

Для подтверждения эффективности и применимости на практике итоговой, 6-й модели спрогнозировали премию в анонсируемой сделке. Оценка премии проводилась в незавершенной сделке, информация о которой была только анонсирована. Соответственно, премия, уплаченная в будущем покупателем продавцу, будет спрогнозирована согласно конечной эконометрической регрессионной модели.

Все факторы, по которым сделка будет выбрана для анализа, остаются неизменными, кроме того, что на момент исследования она не была завершена.

Главная особенность незавершенной сделки проявилась в том, что стоимость, уплаченная в ее ходе (deal value), обозначена лишь оценочно. Стоимость сделки указывается с учетом информации из новостей или фактического значения стоимости акций компании на данный период времени.

Пошагово повторяя схему 1, выполняем первый этап: итак, название рассматриваемой сделки - «Monsanto in talks to acquire Syngenta». Покупатель - американская компания MONSANTO COMPANY, занимающаяся сельским хозяйством, химической и нефтяной промышленностью, пластиком и организацией бизнес-услуг. На данном этапе готовится завладеть $100 \%$ акций швейцарской компании (продавца) SYNGENTA AG, реализующей свою деятельность в химической и нефтяной промышленности. Тип сделки: горизонтальная интеграция, враждебный тип поглощения. Слухи о сделке появились еще 23.06.2014, однако официально о ней объявили лишь 08.05.2015.

Была предпринята попытка оценить возможную величину премии с учетом известных на данный момент обстоятельств.

Второй этап: расчет премии с учетом цены акции за 1 день до объявления о сделке (методика информационной базы Zephyr) показал, что:

Acquisition premium $=34,96 \%$.

Такое высокое значение премии может быть объяснено враждебностью к проведению сделки. Покупатели, желая добиться своих целей, вынуждены платить более высокую цену за акции компа- 
нии-мишени, для того чтобы задобрить акционеров компании. Возможно предположение о том, что если бы сделка была дружественной, то размер выплат за акции был бы намного ниже.

Вышеизложенные предположения могут быть подтверждены тем, что цена за акцию компании${ }_{\Omega}$ мишени за три месяца до анонсирования сделки была равна 342,52 долл. США, а уже на момент ○объявления составила 486,75 долл. США.

-Третий шаг: расчет стоимости компании - мишени с помощью мультипликатора EV/EBITDA.

Необходимые данные для расчета представлены в таблице 18.

Таблицุа 18

Данные для расчета стоимости компании-мишени [на основе данных Zephyr]

\begin{tabular}{|c|c|c|c|}
\hline Показатель & $\begin{array}{c}\text { EV или стоимость компании- } \\
\text { цели на момент сделки } \\
\text { (Target enterprise value USD } \\
\text { Last. avail. year) }\end{array}$ & $\begin{array}{c}\text { EBITDA компании-цели } \\
\text { (Target EBITDA USD Last } \\
\text { avail. year) }\end{array}$ & $\begin{array}{c}\text { Мультипликатор EV/ } \\
\text { EBITDA }\end{array}$ \\
\hline \\
\hline
\end{tabular}

( () Достаточно высокое по сравнению со среднеотраслевым показателем значение мультипликатора $\llcorner\mathrm{EV} / \mathrm{EBITDA}$ говорит о том, что на сегодня складываются не лучшие обстоятельства для инвестора ㅇносительно вложения средств в данный актив.

бККак и в прошлых расчетах, при вычислении стоимости компании-мишени принимают среднеот๓раслевой показатель мультипликатора, который равен 5,09. Bec EV/EBITDA - 1.

文 Справедливая рыночная стоимость компании-мишени, рассчитанная методом компаний-аналогов, ऽравна:

款FMV $V_{\text {к-м }}=5354000000 * 5,09 * 1=27251860000$ долл. США.

Четвертый шаг: оценка величины премии по следующей формуле:

$A P R=\frac{P-F M V}{F M V} \times 100 \%$,

где $A P R \equiv$ премия за сделку, уплаченная компанией-продавцом компании-мишени;

$\mathrm{P} \equiv$ цена, уплаченная инвестором за приобретаемую компанию;

$F M V \equiv$ справедливая рыночная стоимости компании-мишени.

Таблица 19

Данные для расчета премии

\begin{tabular}{|c|c|c|}
\hline Показатель & $\begin{array}{c}\text { Р (цена сделки - deal } \\
\text { value USD) }\end{array}$ & FMV (USD) \\
\hline Величина показателя & 45241058671 & 27251860000 \\
\hline
\end{tabular}

$A P R=66 \%$.

Разница в показателях премий, рассчитанных различным образом, может объясняться различным подходом к оценке стоимости компании-мишени. Так как сделка характеризуется как враждебное поглощение, вполне очевиден тот факт, что переплата за нее будет велика.

Пятый этап: расчет премии с использованием регрессионной модели.

Модель выглядит следующим образом:

ACQ_PREM $=3,58^{*}\left(10^{\wedge}-6\right)$ mark_cap $-3,75^{*}\left(10^{\wedge}-7\right)$ turnover $-4,13^{*}\left(10^{\wedge}-9\right)$ out shares $-7,56^{*}\left(10^{\wedge}\right.$ -7) deal val $+6,2086$ transnat $+6,9849$ inf as $+11,11$ vert int $+11,38 \mathrm{congl}$ int ACQ PREM $=66,6 \%$.

Заключительный этап или сравнение результатов и подведение итогов эффективности модели: полученные результаты можно рассматривать лишь как возможный дальнейший путь развития сдел- 
ки. Акционеры поглощаемой компании могут как еще больше увеличить цену, так и уменьшить ее, если сделка примет дружественный оттенок. Анализируя полученные результаты, можно говорить о том, что показатели премии данной (предполагаемой) и премий рассчитанных различаются, что также говорит о различных методиках и допущениях в расчетах. Но, несмотря на все недостатки, 乞регрессионная модель показала приблизительный результат показателя премии к рассчитанному с ヘпомощью предложенной методики в данной работе показателю.

$\underset{\mathrm{J}}{\mathrm{N}}$ Как известно, для того чтобы компенсировать затраты, полученные в ходе сделки, компания-покупатель должна в будущем компенсировать эти затраты. Чем выше премия, тем больше необходимость резкого увеличения доходности компании-инициатора в будущем. И.В. Ивашковская [Ивашковская, 2007] в своем исследовании «Слияния и поглощения: ловушка роста» утверждает, что при допущении о том, что премия была бы равна $0 \%$, в последующие 10 лет компания-поглотитель должна была бы увеличивать свою доходность на 15\% каждый год. А если премия имела значение $75 \%$, то для дальнейшего существования фирмы, не говоря о каких-либо успехах в ее деятельности, компании надо увеличить свою доходность хотя бы на 101\%, чтобы не стать банкротом в первый же год после сделки. И лишь только через 10 лет компания может остановиться на 22\% увеличения ×доходности в год. Это залог успешного существования компании после завершения сделки М\&А.

Подводя промежуточные итоги, был проведен статистический и эконометрический анализ факторов, которые так или иначе могут влиять на образование показателя премии в сделках слияния и поглощения. Сначала была предпринята попытка построить модель только с участием количебственных показателей, то есть основная гипотеза: финансовые показатели компании-цели влияют

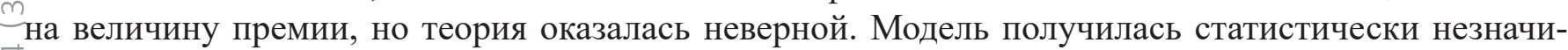
оेмой, не давала эффективной проверки. Поэтому для увеличения объясняющей способности модели × нее были введены некоторые дамми-переменные, то есть фиктивные переменные. Среди них и способ оплаты, и трансграничный тип сделки, и тип интеграции, и оценка конкурентной среды,

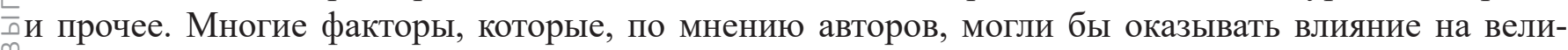
чину премии, не смогли участвовать в построении модели ввиду закрытости информации. После того как финальная модель показала достаточно высокую объясняющую способность, необходимо было апробировать ее на практических данных. Для этого была рассмотрена и проанализирована сделка, совершенная между норвежской и гонконгской компаниями, проведена оценка величины премии различными способами расчетов, а затем величина премии была точечно оценена с помощью регрессионной финальной модели. Несмотря на среднюю эффективность модели, результаты, полученные различными способами, отличались друг от друга на незначимую величину. Подробно результаты описаны после проведения анализа. Финальной проверкой «жизнеспособности» модели была попытка спрогнозировать величину премии в незавершенной сделке. К анализу была применена сделка между американской и швейцарской компаниями. Сначала была рассмотрена вся доступная информация о сделке, затем сделаны некоторые предположения о характеристиках, мотивах, целях, способах и этапах проведения сделки на основе имеющихся доступных данных. С помощью все той же финальной модели была проведена точечная регрессионная оценка величины премии. Показатели величины премии, полученные в результате расчета традиционными методами, и показатель, полученный способом оценки регрессионной модели, оказались очень близки.

Итак, для определения оптимальной премии в сделках слияния и поглощения в нефтегазовом секторе была разработана универсальная эконометрическая модель. Модель выявила следующее влияние факторов на образование величины премии:

- Рыночная капитализация положительно влияет на образование величины премии. Чем выше ее показатель, тем выше премия.

- Операционная прибыль компании-мишени и количество размещенных акций находятся в отрицательной зависимости от величины премии.

- Благодаря модели была выявлена зависимость между типом интеграции и премией: при конгломератных и вертикальных (вертикальных - немного ниже) слияниях величина премии значительно выше, чем при горизонтальном слиянии. Данный факт объясняется тем, что при конгломератном типе слияния чаще всего происходят вражеские поглощения, а по- 
лучить свое и сломить сопротивление со стороны топ-менеджмента компании-цели можно, заплатив высокую премию за сделку акционерам компании.

- Также исследование показало, что информационная асимметрия положительно влияет на премию. Можно предположить, что в неведении покупатель оценит стоимость компании, а соответственно и величину премии, лучше, чем если бы он был знаком с компанией-мишенью ранее. Возможно, присутствие информационной асимметрии исключит предвзятое отношение, а также сделает его оценку объективной.

- $\quad$ Трансграничный тип сделки также оказывает положительное влияние на величину премии.

- Необходимо также отметить, что не только данные финансовые показатели компании-цели оказывают влияние на величину премии, но и связанные с ними показатели, которые могут быть использованы при расчете. На ранних стадиях создания модели многих финансовые факторы, тесно коррелирующие друг с другом, были исключены, чтобы избежать искажения результатов модели. Но очевиден факт, что при оценке величины премии оценщики ориентируются и на них.

Итоговая модель имеет среднюю объясняющую способность. Но все же в отношении тех конкретных сделок, которые были рассмотрены в практической части, модель показала близкие к фактическим значениям результаты. По предположению авторов, это может быть связано с тем, что в модели не были учтены некоторые факторы, которые являются значимыми в фундаментальных исследованиях. Ввиду отсутствия информации о многих финансовых показателях и мультипликаторах компании-цели большой процент сделок был исключен из исследования, как и некоторые факторы, чтобы избежать удаления такого количества сделок, которое оказало бы влияние на про일ㄹение исследования. Также ввиду отсутствия такой информации в открытом доступе не было возможности включить показатели, касающиеся предположительной синергии в будущем, как опеЕрационной, так и финансовой. Данные о синергии от устранения неэффективного управленческоळ $г$ аппарата также не имели никаких показателей в базе. Наличие или отсутствие конкурентов у компании-покупателя также не удалось определить. Только в информации и единицах сделки были данные такого рода. Их пришлось исключить ввиду нецелесообразности использования малого количества показателей.

В заключение необходимо сказать, что улучшить модель можно увеличением количества наблюдений, то есть сделок, а также расчета и поиска информации и данных по показателям, на которые сложно дать однозначный ответ без проведения подробного анализа.

\section{Список литературы:}

1. Ивашковская, И.В. Моделирование стоимости компании. Стратегическая ответственность совета директоров. М.: ИНФРА-М, 2009.

2. Ивашковская, И.В. Слияния и поглощения: ловушки роста // Управление компанией. 2007. № 4 C. 26-29.

3. Рынок слияний и поглощений в 2013 году. Выпуск ежегодного исследования KPMG «Рынок слияний и поглощений в России в 2013 году». [Электронный ресуpc] URL:: http:/www. kpmg.com/RU/ru/IssuesAndInsights/ArticlesPublications/Documents/S_MA_2r_2013.pdf (дата обращения: 15.03.2015).

4. Рынок слияний и поглощений в 2014 году. Выпуск ежегодного исследования KPMG «Рынок слияний и поглощений в России в 2014 году». [Электронный ресурc] URL: http:/www.kpmg. com/RU/ru/IssuesAndInsights/ArticlesPublications/Documents/S_MA_4r_2015.pdf (дата обращения: 01.04.2015).

5. Elgers, P. and Clark, J.(1980), "Merger Types and Shareholder Returns: Additional Evidence", Financial Management, Vol. 4, № 2, pp. 66-72.

6. Gupta, D. and Gerchak, Y.(2002), "Quantifying Operational Synergies in a Merger / Acquisition", Management Science, Vol. 48, № 4, pp. 517-533.

7. Malatesta, P. (1983), "The Wealth Effect of Merger Activity and the Objective Functions of 
Merging Firms", Journal of Financial Economics, Vol. 11, pp. 155-181.

8. Manne, H. (1965), "Mergers and the Market of Corporate Control”, Journal of Political economy, Vol. 73, pp. 110-120.

9. Nielsen, J.F., and Melicher, R.W. (1973), “A Financial Analysis of Acquisition and Merger Premiums", The Journal of Financial and Quantitative Analysis, Vol. 8, № 2, pp. 139-148.

10. Rose, P.(1987), "The Impact of Merges in Banking”, Journal of Economics and Business, Vol. 39, pp. 289-312.

11. Sirower, M. (1997), "The Synergy Trap: How Companies Lose the Acquisition Game", The Free Press, Vol. 2, № 1.

12. Slusky, A., and Caves, R. (1991), "Synergy, Agency, and the Determinants of Premia Paid in Mergers", The Journal of Industrial Economics, Vol. 39, № 3, pp. 277-296.

13. Smith, K.W., and Triantis, A. (2004). The Value of Options in Strategic Acquisitions. In Real Options in Capital Investment: Models, Strategies and applications, ed. L. Trigeorgis. Westport, Conn.: Praeger.

14. Walkling, R., Edmister, R. (1985), "Determinants of Tender Offer Premiums", Financial Analysts Journal, Vol. 41, № 1, pp. 27-37.

15. Wansley, J.W., Lane, W.L., and Yang, H.C.(1983), “Abnormal Returns to Acquired Firms by Type of Acquisition and Method of Payment”, Financial Management, Vol. 12, № 3, pp. 16-22.

16. Varaiya, N.P. (1987), "Determinants of Premiums in Acquisition Transactions", Managerial and Decision Economics, Vol. 8, № 3, pp. 175-184.

17. Zephyr Annual M\&A Report Global, 2014 // Bureau van Dijk. Available at: www.bvd.co.uk/ zephyrreport/GlobalFY2014.pdf [Accessed: 30th April 2015].

18. Zephyr Week in Review, 30th March 2015 // M\&A Portal. Available at: http://www.mandaportal. com/getattachment/91a42d93-28f6-46e8-9b41-80c1e8788cd/Zephyr-Week-in-Review,-30thMarch-2015 [Accessed: 1th April 2015]. 
DETERMINATION OF AN OPTIMUM PREMIUM PAID
IN M\&A TRANSACTIONS IN OIL AND GAS SECTION

\section{IN M\&A TRANSACTIONS IN OIL AND GAS SECTION}

Researcher, Associate Professor, National Research University

Higher School of Economics,

Oxana Shevyakina,

National Research University Higher School of Economics, Russia

Abstract

Mergers and acquisitions are an important part of the economy of any country. In difficult circumstances companies increasingly difficult to carry out a successful deal M \& A. It is necessary to take into account the large number of nuances in preparation for the transaction, which have a direct impact on the quality of its implementation. One of the most important nuances is to determine the optimal value of the premium ${ }^{\circ}$ in the transaction $M \&$ A. This issue contains a very sound basis for understanding the true direction of the transaction $\mathrm{M} \& \mathrm{~A}$, since it affects, it is advantageous to be performed dealings, whether a positive syn(-) ergistic effect and many other important aspects. Generally speaking, if a company - not just the absorber $\underset{\sim}{\sim}$ actually calculate the correct value of the premium, it may be at a loss for a deal giving a greater value $\checkmark$ than it receives from the profit.

6 The thematic justification of this study is that at the planning stage of M \& A transactions is necessary to

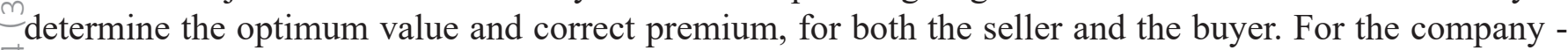
olseller, it becomes an important aspect of the deal to receive an amount not less than the value of the part $r$ of the assets which he sells. For the buyer it is important not to overpay for the deal because a too high $\geq$ premium guarantee an absent of future synergies, and possibly negative outcome for the company until its bankruptcy.

The purpose of this article focuses on the determination the formation of the optimal value premium in $\mathrm{M}$ \& A transactions, in terms of the oil and gas sector, through the identification of financial and non-financial factors that have a direct impact on the premium rate.

The object of this study is M \& A transactions in the oil and gas industry. Item - premiums paid during the M \& A transactions in the oil and gas sector by the buyer to the seller.

In consequence of research, the regression model was developed. This model explaining the dependence of the acquisition premium value's of certain financial and non-financial factors: the market capitalization has a positive effect on the formation of the value of the premium; operating profit target and the number of outstanding shares are negative depending on the size of the premium; at the conglomerate and vertical (vertical - slightly below) mergers the premium size is significantly higher than the horizontal merger; information asymmetry has a positive effect on the premium; cross-border transaction type also has a positive effect on the value of the premium. The final model has a strong explanatory power.

It is necessary to be noted that not the only financial data of the financial performance of the target is influenced on the amount of premiums but some related factors which may be used in calculating each other in this type of research.

Keywords: mergers and acquisitions, company's value, acquisition premium, oil and gas sector, econometric regression multivariable model

JEL: L1

\section{References}

1. Ivashkovskaja, I.V. (2009), Modelirovanie stoimosti kompanii. Strategicheskaja otvetstvennost' soveta direktorov, INFRA-M, M.

2. Ivashkovskaja I.V. (2007)/ “Slijanija i pogloshhenija: lovushki rosta”. Upravlenie kompaniej. №4, pp.26-29 
3. Rynok slijanij i pogloshhenij v 2013 godu // Vypusk ezhegodnogo issledovanija KPMG «Rynok slijanij i pogloshhenij v Rossii v 2013 godu».. Available at: http://www.kpmg.com/RU/ru/IssuesAndInsights/ArticlesPublications/Documents/S_M A_2r_2013.pdf (data obrashhenija: 15.03.2015).

4. Rynok slijanij i pogloshhenij v 2014 godu // Vypusk ezhegodnogo issledovanija KPMG «Rynok slijanij i pogloshhenij v Rossii v 2014 godu». Available at: http://www.kpmg.com/RU/ru/IssuesAndlnsights/ArticlesPublications/Documents/S_MA_4r_2015.pdf (data obrashhenija: 01.04.2015

5. Elgers, P. and Clark, J.(1980), "Merger Types and Shareholder Returns: Additional Evidence", Financial Management, Vol. 4, № 2, pp. 66-72.

6. Gupta, D. and Gerchak, Y. (2002), "Quantifying Operational Synergies in a Merger / Acquisition”, Management Science, Vol. 48, № 4, pp. 517-533.

7. Malatesta, P. (1983), "The Wealth Effect of Merger Activity and the Objective Functions of Merging Firms", Journal of Financial Economics, Vol. 11, pp. 155-181.

8. Manne, H. (1965), "Mergers and the Market of Corporate Control”, Journal of Political economy, Vol. 73, pp. 110-120.

9. Nielsen, J.F. and Melicher, R.W. (1973), “A Financial Analysis of Acquisition and Merger Premiums", The Journal of Financial and Quantitative Analysis, Vol. 8, №. 2, pp. 139-148.

10. Rose, P. (1987), "The Impact of Merges in Banking”, Journal of Economics and Business, Vol. 39, pp. 289-312.

11. Sirower, M. (1997), "The Synergy Trap: How Companies Lose the Acquisition Game", The Free Press, Vol. 2, № 1.

12. Slusky, A. and Caves, R. (1991), "Synergy, Agency, and the Determinants of Premia Paid in Mergers", The Journal of Industrial Economics, Vol. 39, № 3, pp. 277-296.

13. Smith, K.W., and Triantis, A. (2004), The Value of Options in Strategic Acquisitions. In Real Options in Capital Investment: Models, Strategies and applications, ed. L. Trigeorgis. Westport, Conn.: Praeger.

14. Walkling, R., Edmister, R. (1985), "Determinants of Tender Offer Premiums", Financial Analysts Journal, Vol. 41, № 1, pp. 27-37.

15. Wansley, J.W., Lane W.L., Yang H.C.(1983), “Abnormal Returns to Acquired Firms by Type of Acquisition and Method of Payment”, Financial Management, Vol. 12, № 3, pp. 16-22.

16. Varaiya, N.P. (1987), "Determinants of Premiums in Acquisition Transactions", Managerial and Decision Economics, Vol. 8, № 3, pp. 175 - 184.

17. Zephyr Annual M\&A Report Global, 2014 // Bureau van Dijk. Available at: www.bvd.co.uk/ zephyrreport/GlobalFY2014.pdf [Accessed: 30th April 2015].

18. Zephyr Week in Review, 30th March 2015 // M\&A Portal. Available at: http://www.mandaportal. com/getattachment/91a42d93-28f6-46e8-9b41-80c1e8788cd/Zephyr-Week-in-Review,-30thMarch-2015 [Accessed: 1th April 2015]. 